\title{
Geochemical characteristics of Proterozoic granite magmatism from Southern Granulite Terrain, India: Implications for Gondwana
}

\author{
T YELlAPPA* and J MALLIKHARJUnA RAO \\ National Geophysical Research Institute (Council of Scientific and Industrial Research), Uppal Road, \\ Hyderabad 500 00\%, India. \\ ${ }^{*}$ Corresponding author. e-mail: yellappa_thoti@yahoo.co.uk
}

MS received 20 November 2016; revised 12 July 2017; accepted 14 July 2017; published online 6 March 2018

Granitoid intrusions occur widely in the Southern Granulite Terrain (SGT) of India, particularly within the Cauvery Suture Zone (CSZ), which is considered as the trace of the Neoproterozoic Mozambique ocean closure. Here we present the petrological and geochemical features of 19 granite plutons across the three major tectonic blocks of the terrain. Our data show a wide variation in the compositions of these intrusions from alkali feldspathic syenite to granite. The whole rock geochemistry of these intrusions displays higher concentrations of $\mathrm{SiO}_{2}, \mathrm{FeO}^{*}, \mathrm{~K}_{2} \mathrm{O}$, Ba, Zr, Th, LREE and low $\mathrm{MgO}, \mathrm{Na}_{2} \mathrm{O}, \mathrm{Ti}, \mathrm{P}$, $\mathrm{Nb}, \mathrm{Y}$ and HREE's. The granitoids are metaluminous to slightly peraluminous in nature revealing both I-type and A-type origin. In tectonic discrimination plots, the plutons dominantly show volcanic arc and syn-collisional as well as post-collisional affinity. Based on the available age data together with geochemical constrains, we demonstrate that the granitic magmatism in the centre and south of the terrain is mostly associated with the Neoproterozoic subduction-collision-accretion-orogeny, followed by extensional mechanism of Gondwana tectonics events. Similar widespread granitic activity has also been documented in the Arabian Nubian shield, Madagascar, Sri Lanka and Antarctica, providing similarities for the reconstruction of the crustal fragments of Gondwana supercontinent followed by Pan-African orogeny.

Keywords. Southern granulite terrain; Cauvery suture zone; I-type and A-type granites; Neoproterozoic subduction; Gondwana.

\section{Introduction}

Granitoid intrusions represent a large part of the continental crust and occur in different tectonic environments (Pitcher 1993). The generation and emplacement of granitic magmas in space and time represent a major contribution to the growth and recycling of continental crust (Castro et al. 1999a; Patino Douce 1999). In general, the crustal melting and mixing of mantle are the major processes that generate granitoids (Clark et al. 1997). The genetic classification of granites is based on the nature of origin considering those derived from sedimentary protolith as S-type, igneous protoliths as I-type, recycled and hydrated continental crust as A-type, and those evolved directly from subducted oceanic crust or overlying mantle as M-type (Chappell and White 1974; Collins et al. 1982; Whalen et al. 1987). Based on their tectonic environment, granitoids have been classified as island arc 
granitoids (IAG); continental arc granitoids (CAG); continental collision granitoids (CCG); postorogenic granitoids (POG); rift-related granitoids (RRG); continental epirogenic uplift granitoids (CEUG); and oceanic plagiogranites (OP). Among these, the IAG, CAG, CCG, and POG are considered as orogenic granitoids, whereas the RRG, CEUG and OP are considered as anorogenic granitoids (Maniar and Piccoli 1989). Granitic magmatism usually spreads over a wide range of settings including volcanic areas, continental shields and orogenic belts. It has been well established that during orogenesis, dynamic melting of anisotropic crust due to differential stresses leads to heterogeneous deformation which enables granite extraction, ascent and emplacement (e.g., Brown 1994; Sawyer 1994; Collins and Sawyer 1996; Rushmer et al. 1998). Granitoid formations associated with major collisional events have been traced around the world since Archean to Phanerozoic (Burg and Ford 1997). The Neoproterozoic Pan-African orogeny (850-550 Ma; Kroner 1980; Shackelton $1986)$ is one of the events where large-scale tectonics of continental convergence, deformation, metamorphism and granitic magmatic activity occurred within the Gondwana crustal fragments including South America, Africa, Australia, India and Antarctica (Kusky et al. 2003; Kroner and Stern 2004). This magmatism mainly represented by granites, alkaline granites and syenites is widely reported particularly from the Eastern Gondwana continents, i.e., India, Sri Lanka, Madagascar, East Antarctica and Western Australia (Rajesh et al. 1996; Collins 2003; Jacobs et al. 2003; Ghosh et al. 2004; Santosh et al. 2005). In India, the central domain of the Southern Granulite Terrain has witnessed widespread felsic alkaline magmatism during the Neoproterozoic period (Nathan et al. 2001; Ghosh et al. 2004). In addition, there are several Neoarchean-Paleoproterozoic granitoid intrusions, charnockites related magmatic suites occur in several parts of the terrain (Ghosh et al. 2004; Clark et al. 2009; Saitoh et al. 2011). The Neoproterozoic granitoids are mostly restricted to central and southern parts of Tamil Nadu and Kerala states. Further, several intrusions have occurred along the E-W trending Cauvery Suture Zone (CSZ) and towards its north as well as to its south as described by several workers (e.g., Gopalakrishnan 1994). Previous studies have correlated this magmatism to Archean crustal thickening (Drury et al. 1984), Precambrian continent-continent collision and southward subduction of Dharwar craton
(Rai et al. 1993) and crustal recycling. Based on isotopic ages of several granitic rocks from southern India, it is defined as two contrasting age provinces, the northern Dharwar craton of Archean age and the southern mobile belt of Proterozoic age with the CSZ marking the boundary between the two blocks (Harris et al. 1994). Some of the granitic rocks occur near the transition zone around Krishnagiri in the northern part of Tamil Nadu ranging in age from 3400 to 1000 Ma (Monard 1983) and have been classified into I-type and S-type (Condie et al. 1982, 1986; Dhanaraju et al. 1983; Chandrasekharan 2002). Whereas many of the granitoids occurring within the CSZ and south of the CSZ are of Neoproterozoic age. Recently, it is also described that the Mesoarchean granitoids of $3184.0 \pm 5.5 \mathrm{Ma}, 3170.3 \pm 6.8 \mathrm{Ma}$ (Santosh et al. 2015) and also the occurrence of Hadean felsic continental crust in the northwestern part of the terrain within the Coorg Block (Santosh et al. 2016). In this paper, we present the mean geochemical data of 19 granitic plutons, particularly of SGT in the three different tectonic blocks including Northern Block of the terrain, CSZ and Madurai Block (figure 1) and evaluated their petrogenesis and tectonic implications for Gondwana supercontinent.

\section{Geological setting}

The SGT in India is one of the largest exposed Precambrian deep continental crustal sections in southern India, consisting of multiple deformed Archean and Neoproterozoic high-grade metamorphic and magmatic rocks. The important lithologies comprise tonalitic gneisses, migmatites with high-grade assemblages of garnet-bearing felsicmafic granulites, charnockites, dismembered mafic and ultramafic rocks, magnetite-rich quartzites and granitoids. The terrain has been divided into a number of distinct crustal blocks based on the structural and isotopic evolution from the north to south: (1) the Northern Block, (2) the Nilgiri Block, (3) the Salem-Madras Block, (4) Cauvery Suture Zone (CSZ), (5) the Madurai Block, and (6) the Trivandrum Block, (figure 1; Naqvi and Rogers 1987; Santosh 1996; Bartlett et al. 1998; Chetty and Bhaskar Rao 2006; Ramakrishnan and Vaidyanathan 2008; Clark et al. 2009; Santosh et al. 2009; Plavsa et al. 2012; Collins et al. 2014). The Northern Block is described below the 'Fermor line' and is dissected into three major blocks 


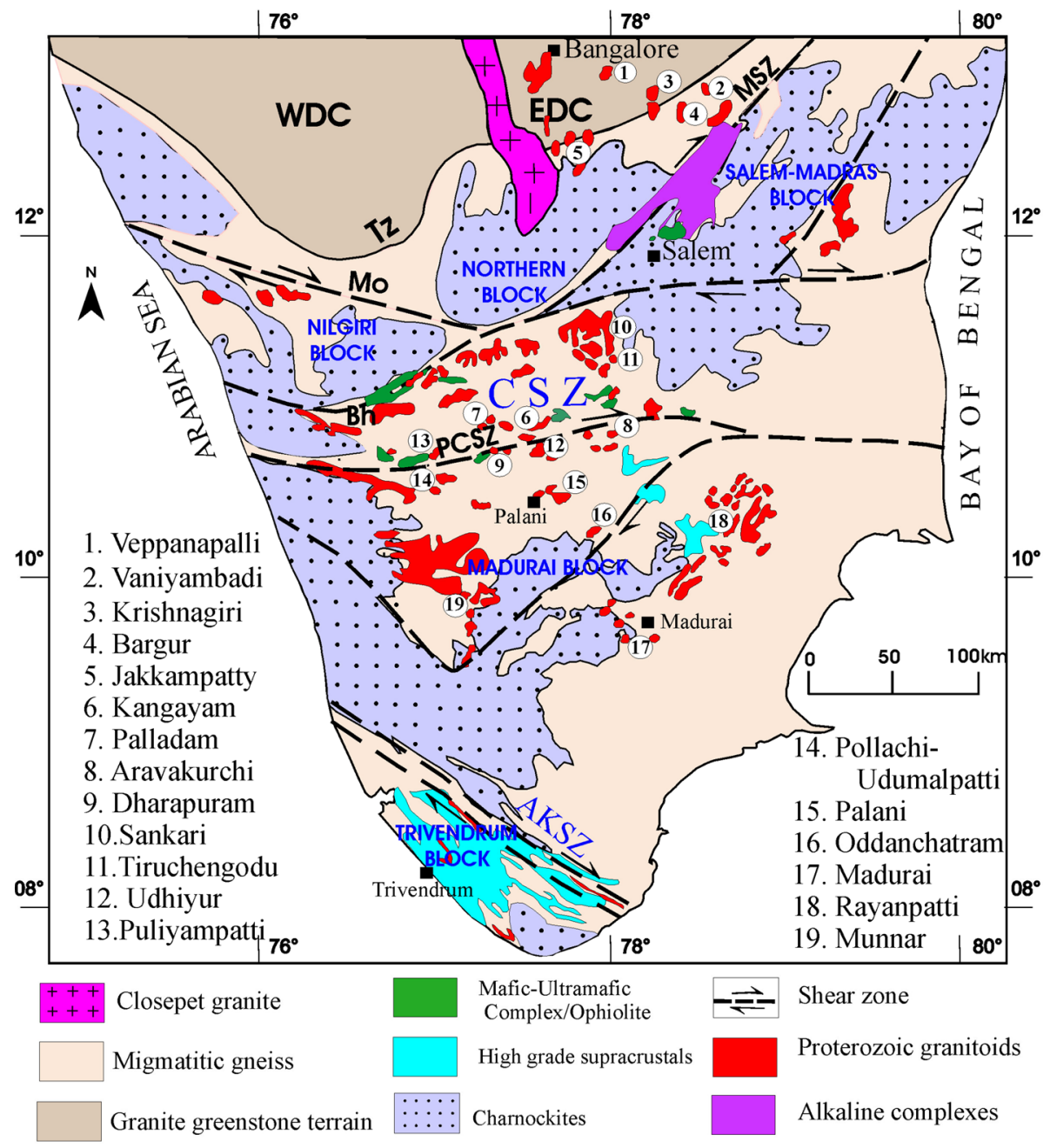

Figure 1. Geological map of southern India showing the distribution of granite plutons in major tectonic blocks (modified after Santosh and Sajeev 2006 and source from Geological Survey of India maps). Shear Zones. M: Moyar, Bh: Bhavani, Me: Mettur, Pa-Ca: Palghat-Cauvery, Ga: Gangavalli CSZ: Cauvery Suture Zone, AKSZ: Achankovil Shear Zone, WDC: Western Dharwar Craton, EDC: Eastern Dharwar Craton, Tz: Transition Zone. Location of the granite plutons are also shown in the map.

by a central zone of faults and ultrabasic-alkaline magmatism (Ramakrishnan 2003). The block consists of massive charnockites that are interbedded with supracrustals, bands of fuchsite-kyanitesillimanite quarzites, cordierite-sillimanite-kyanite schist, calc-granulites, limestones, several bands of amphibolites and banded iron formations. Several bodies of ultrabasic-alkaline-syenite-carbonatite complexes and granitoids have also emplaced within hornblende gneisses (Gopalakrishnan 1994; Ramakrishnan 2003). The Nilgiri Block, which occurs northwest of the terrain, predominantly consists of strongly deformed and migmatized garnetiferous rocks, enderbitic granulites, charnockite massifs with minor contributions of kyanite-bearing gneisses, alkali granites, quartzites and metabasites
(Peucat et al. 1989; Janardhan et al. 1994) that form highland areas interspersed with low-lands consisting of felsic rocks generally in amphibolite facies. The Salem-Madras Block occurs in northeast of the terrain and consists of orthogneiss, charnockite, mafic granulite and ultramafic intrusions in association with metasedimentary units, a succession that continues up to the PalghatCauvery Shear Zone (PCSZ), and the entire zone is termed as Cauvery Suture Zone and is considered as the trace of the Cambrian suture zone of Gondwana (Collins et al. 2007). Based on the $\mathrm{U}-\mathrm{Pb}$ SHRIMP ages from charnockites of SalemMadras Block, Clark et al. (2009) described the magmatism at ca. 2530 Ma age and subsequent high-grade metamorphism and partial melting at 
ca. 2480 Ma. The available geochronological data on the protoliths of the Salem Block indicate Mesoto Neoarchean rocks stretching as far south as the PCSZ, with metamorphic ages indicating a granulite facies event in the latest Archean to early Proterozoic times (Peucat et al. 1993). The Salem Block also represents the southernmost extent of the Archean Dharwar craton, which is separated from the Archean to Proterozoic Madurai Block by the Cauvery Suture Zone (CSZ, figure 1). The CSZ is made up of Neoarchean and Neoproterozoic oceanic type subduction complexes including ophiolitic melanges, high-pressure eclogites and ultra-high temperature sapphirine bearing rocks, mafic ultramafic complexes, charnockites, two pyroxene granulites, island-arc volcanic rocks, quartzo-feldspathic hornblende gneisses with several events of granite (Viswanathan et al. 1990; Gopalakrishnan 1994; Hussain et al. 1996; Santosh et al. 2009, 2013; Yellappa et al. 2010, 2012). The CSZ extends from west coast to east coast with a distance of about $400 \mathrm{~km}$ and a width of $\sim 60 \mathrm{~km}$ and comprises a network of shear zones. Chetty et al. (2003) described several shear networks of CSZ including Moyar-Bhavani Shear Zone (MBSZ), the Chennimalai-Noyil Shear Zone (CNSZ), the Dharapuram Shear Zone (DSZ), the Devattur-Kallimandayam Shear Zone (DKSZ) and the Karur-Oddanchatram Shear Zone (KOSZ) in the central part of the CSZ. While the MoyarBhavani-Attur Shear Zone represents the northern margin, the Palghat-Cauvery Shear Zone marks the southern margin. There are three ophiolitic complexes that have been described recently from the CSZ; (i) Neoproterozoic Manamedu Ophiolite Complex (MOC), occurring at the northern bank of the Cauvery river course, $40 \mathrm{~km}$ south east of Namakkal, (ii) Devanur Ophiolitic Complex (DOC) of Neoarchean age (Santosh et al. 2012; Yellappa et al. 2012) located about $20 \mathrm{~km}$ north of MOC, and (iii) Neoarchean Agali Ophiolite Complex (AOC), that has been reported from the Agali hill near Attappadi, along the western extension of the CSZ/Bhavani Shear Zone (Santosh et al. 2013). The entire rock sequence of this Agali complex has been interpreted to represent a typical 'Ocean Plate Stratigraphy' sequence with arc and exhumed sub-arc mantle material (Santosh et al. 2013). Recently, Yellappa et al. (2014) also described the mafic and ultramafic complex of Aniyapuram near Mohanur as a dismembered ophiolite suite of Neoarchean to Paleoproterozoic age (Koizumi et al. 2014). All these ophiolite complexes were evolved under two major events of suprasubduction zone tectonics ranging from Neoarchean to Neoproterozoic periods.

The Madurai Block/Madurai Granulite Block (MGB) occurs immediately south of the CSZ and is the largest crustal block in southern India. This block comprises dominantly of charnockite massifs intercalated with tonalitic/granodioritic gneisses and elongated narrow belts and slivers of metasedimentary rocks including quartzites, metamorphosed carbonates, iron formations and pelites, all suggesting an accretionary realm (Santosh et al. 2009). A N-S trending Karur-Kambam-PainavuTrissur Shear Zone (KKPT) runs within the centre of MGB (Ghosh et al. 1998, 2004). The MGB can be lithologically divided into a western region and an eastern region (Cenki and Kriegsman 2005). The western part is characterized by two different groups of hornblende-biotite and orthopyroxenebiotite (charnockite) gneisses, one being quartz rich and the other is feldspar-rich rock, and alkali granites. The eastern part is composed of massive charnockites and enderbites with heterogeneously distributed quartzites and calc-silicate series of rocks (Cenki and Kriegsman 2005). The southern boundary of the Madurai Granulite Block is marked by the Achankovil Shear Zone (AKSZ), which also separates the Madurai Block from the Trivandrum Block in the south. The Trivandrum Block is sub-divided on lithological grounds into three tectonic units: the Kerala Khondalite Belt (KKB), the Nagercoil unit and the Achankovil metasediments. Trivandrum Block comprises dominantly of metasedimentary gneisses including garnet-bearing felsic gneisses (known locally as leptynites), orthopyroxene, hornblende, and biotite ( \pm garnet)-bearing intermediate to acid granulites of broadly granitic composition (charnockites to enderbites), granulite facies garnet+spinel+cordierite+sillimanite metapelites (termed khondalites) and garnet-bearing quartzo feldspathic genisses interlayered with charnockites (Rajesh 2004; Ramakrishnan and Vaidyanathan 2008). Calc-granulites, quartzites and ultramafic rocks also occur as linear bodies parallel to the local structural trends. These lithologies constitute a vast sequence of continental margin sediments originally defined as the Kerala Khondalite Belt (KKB; Chacko et al. 1987). Achankovil Shear Zone (AKSZ) holds a key position in juxtaposing the member terrains in eastern Gondwana supercontinent and has been correlated with the sinistral Ranostara Shear Zone of Madagascar (e.g., Ramakrishnan 1991; 
Paquette et al. 1994; Windley et al. 1994; Kriegsman 1995). Garnet- and biotite-bearing leucogranites are abundant throughout the KKB and formed from high-T dehydration-melting of the various $\mathrm{KKB}$ gneisses at granulite-facies conditions subsequent to the main stage of Pan-African ductile deformation. Neoproterozoic magmatic events and occurrences of 590-560 Ma age of granite within the shear zone have also been reported from AKSZ by several workers (Rajesh et al. 1996; Bohm et al. 2003; Santosh et al. 2005; Braun 2006).

\subsection{Granitic magmatism}

The southern granulite terrain was episodically active in the Neoproterozoic to early Paleozoic times with acid magmatism represented by granites spreading across the tectonic boundaries (Rajesh et al. 1996; Ghosh et al. 2004). The Neoarchaean to Paleoproterozoic granitoids mostly confined to Northern Block are Tiruttani, Sholingar, Bisanattam, Ebbari and Krishnagiri (2500 Ma; GSI 1978; Krogstad and Hanson 1988; Peucat et al. 1993). Some of the granites including Veppanapalli, Vaniyambadi, Krishangiri, Bargur and Jakkampatty from this block also occur similar to the above granites. In Salem-Madras Block, there are several occurrences of Neoarchean and Neoproterozoic granites. Metamorphosed granite of Salem, Kanjimalai, Tiruvannamalai and Tirukovilur granites are of Neoarchean to Paleoproterozoic in age, and are mostly associated with peninsular gneiss and migmatitic complex. It is also described that some of the granites at Kullampatti and younger granites of Chalk Hills belongs to Neoproterozoic age. In the CSZ there are several occurrences, some important plutons of Neoproterozoic granites such as Kangayam, Palladam, Aravakurchi, Dharapuram, Sankari, Tiruchengodu, Karamadai, Udhiyur, Puliyampatti and Kabilarmalai (figure 1). Many of these intrusions are small $\left(<20-40 \mathrm{~km}^{2}\right.$ exposures) and trending $\mathrm{E}-\mathrm{W}$ or $\mathrm{NW}-\mathrm{SE}$, in the form of elongated elliptical bodies emplaced within the older migmatized felsic gneisses and charnockites. There are two phases of these varieties: a leucocratic medium-grained pegmataoidal granitic phase of Sankari-Tiruchengodu, and Puliyampatti coarse-grained pink pegmatoidal granites of Karamadai and Marudamaili whose composition ranges from alkali granite to quartzmonzonites (Nathan et al. 1994). In general, many of the granites from the CSZ are medium- to coarse-grained and contain various amounts of mafic minerals, biotite and hornblende along with felsic minerals, and are mostly granodioritic to monzogranitic in composition with few granites (e.g., Pollachi-Udumalpet) belong to syenite, syenodiorite and monzogranite to tonalitic varieties (Mallikharjuna Rao et al. 2005). The Peralimala, Ezhimala, Kalpatta and Ambalavayal are some of the granites of Neoproterozoic age described in Nilgiri Block emplaced within the high-grade rocks and these are mostly in alkaline nature (Santosh et al. 1986, 1989; Miller et al. 1996; Rajesh 2008). In the Madurai Block, the Neoproterozoic granites include Palani, Oddanchatram, Madurai, Munnar, Pudukkotai, Usilimpatti, Roayanpatti, Vanjinagarm, Melur, Anagadimonar (Santosh et al. 1986, 1989; Nathan et al. 2001). All these granites are calc-akaline to alkaline in nature and emplaced within the migmatitic gneiss/ enderbitic gneiss and charnockitic association. In Trivandrum Block, similar Neoproterozoic granites like Kalipara granite near Pathanamthitta, Athiringal, Pathanapuram and Chenganoor (Santosh and Drury 1988; Rajesh and Santosh 1996) also occur. Though, the granite plutons are restricted to major tectonic segments, there are some transitional occurrences across the major tectonic boundaries including the intrusions at Salem, Nammakal, Karur and Mettupalliyam areas.

\section{Petrography}

The granites described in this study fall into three tectonic blocks. Northern Block represented by Veppanapalli, Vaniyambadi, Krishnagiri, Bargur and Jakkampatty plutons; the CSZ represented by Puliyampatti, Tiruchengodu, Sankari, Udhiyur, Pollachi-Udumalpet, Kangayam, Palladam, Aravakurchi and Dharapuram granites; and Madurai Block represented by Palani, Oddanchatram, Madurai and Munnar granites. In general, all the granites are medium- to coarse-grained and contain quartz, feldspar, biotite and hornblende. The K-feldspar is either microcline or orthoclase with or without perthitic textures together with subordinate plagioclase. Marginal granulations and rare developments of garnet and myrmekite intergrowths are also noticed. Quartz occurs as large anhedral grains with undulatory extinction, as recrystallized grains and as inclusions in other minerals like feldspars, hornblende and biotite. 
The granites in the Northern Block show distinct mineral and textural variations compared to those in the CSZ and Madurai block. In Veppanapalli granites, K-feldspar is the dominant mineral (44 vol.\%) together with plagioclase (23 vol.\%), hornblende $(4$ vol. $\%)$ and opaques $(2$ vol. $\%)$. The modal compositions (table 1) suggest that the rocks vary from alkali granite to granite (figure 3 ). The Vaniyambadi granites are coarse-grained with perthitic K-feldspar (42 vol.\%; figure 2a) and plagioclase (32 vol.\%). Muscovite and quartz also occur around feldspar grains and secondary sphene, epidote, chlorite and biotite are seen in the groundmass (figure $2 \mathrm{~b}$ ). Modal percentages classify them as alkali granites (figure 3). The Krishnagiri granites are grey to light pink in colour and are coarse-grained with plagioclase as the dominant mineral (37 vol.\%) followed by K-feldspar (32 vol.\%). The K-feldspars are either perthite or microcline perthite and some of these minerals occur as inclusions in plagioclase (figure 2c). Alteration effects are common and fine-grained matrix of quartz-biotite-opaque is developed along grain boundaries. Bent cleavages and bent twin lamellae in plagioclase are well developed and strong undualtory extinction in feldspars and mafic minerals are common, which suggest that the deformation is subsequent to the emplacement. Modal compositional plot of $\mathrm{Q}-\mathrm{A}-\mathrm{P}$ diagram (figure 3 ) classifies the rocks as granites. The Bargur granites are medium- to fine-grained and show gneissic texture. The essential minerals are plagioclase (48 vol.\%) followed by K-feldspar (24 vol.\%), with no perthitic textures, although myrmekitic intergrowth textures are common. Biotite (4.5 vol.\%) is the main mafic mineral and secondary epidote is seen in some deformed rocks. The Jakkampatty granites are coarse-grained with plagioclase (43 vol.\%) and K-feldspars (26 vol.\%).

In the CSZ, Kangayam, Palladam, Aravakurchi, and Dharapuram granites are light pink to grey in colour and vary from medium- to coarse-grained with well-developed hypidomorphic textures. These granite plutons are plagioclase poor (7-23 vol.\%) and rich in K-feldspar (46-66 vol.\%), which is mostly microcline and occasionally show perthitic texture. Biotite is the main mafic mineral (figure 2d). Modal classification suggests that these rocks are mainly syno-granites with marginal affinity to monzogranites. The Tiruchengodu-Sankari granites show no deformational effects except for the margins at few locations. They consists of dominantly K-feldspar (46.5 vol.\%) with subordinate plagioclase (31 vol.\%). The K-feldspar occurs in two generations represented by microcline or microcline perthites. Thin rims of clear albite occur around the microcline (figure 2e). Biotite and muscovite are the accessory minerals along with opaques. The Puliyampatti granites are leucocratic, medium- to coarse-grained and poor in mafic minerals. They contain plagioclase (25-35 vol.\%), K-feldspars (31-50 vol.\%), quartz (17-28 vol.\%) and mafic minerals (1-5 vol.\%). The K-feldspar is mostly microcline and shows myrmekitic texture (figure 2f) developed between plagioclase, quartz and K-feldspar. The PollachiUdumalpet granites are coarse-grained and unaltered and are dominantly composed of perthitic K-feldspar (41-63 vol.\%), plagioclase (5-25 vol.\%), quartz (7-38 vol.\%) and opaques (0.4-4.6 vol.\%). Mafic minerals are hornblende (1-7 vol.\%) and biotite (0.3-7.5 vol.\%) with secondary chlorite, sphene, calcite, epidote and allanite occurrence (figure $2 \mathrm{~g}$ ). Sphene corona structures are developed around opaque minerals and rare garnet is also found.

In the Madurai Block, the granites show some minor variations. The Madurai granites are medium- to coarse-grained and grey in colour with strongly developed gneissosity. K-feldspar is the dominant mineral represented by microcline or microcline perthite (62-66 vol.\%), with modal composition (figure 3) ranging from granite to syenite. Different types of perthites including string, bleb and briad types are common (figure $2 \mathrm{~h}$ ). Plagioclase (12.5 vol.\%) is less compared to other granites in the region. Both hornblende and biotites occur as mafic minerals in these rocks. The granites are poor in plagioclase $(9 \mathrm{vol} . \%)$ and dominated by microcline perthites (65 vol.\%). Palani granites are coarse-grained with K-feldspar, anti-perthitictic and plagioclase is higher than K-feldspar. Biotite is the main mafic mineral with accessories of apatite and secondary epidote. Oddanchatram and Rayanpatti granites are light pinkish in colour with $\mathrm{K}$-feldspar dominating and plagioclase is very less. Perthitic texture with stringe type is common. Clionpyroxene, biotite, zircon, apatite and secondary chlorite are other minerals in these rocks. The Munnar granites are rich in K-feldspars (67-73 vol.\%) and are represented by microcline perthites. Plagioclases is very less (3.5 vol.\%) and mafic minerals are hornblende and biotite. The Munnar granites and Oddanchatram granite plot in alkali feldsphatic syenite field and Rayanpatti granite plots in syenite field (figure 3 ). 


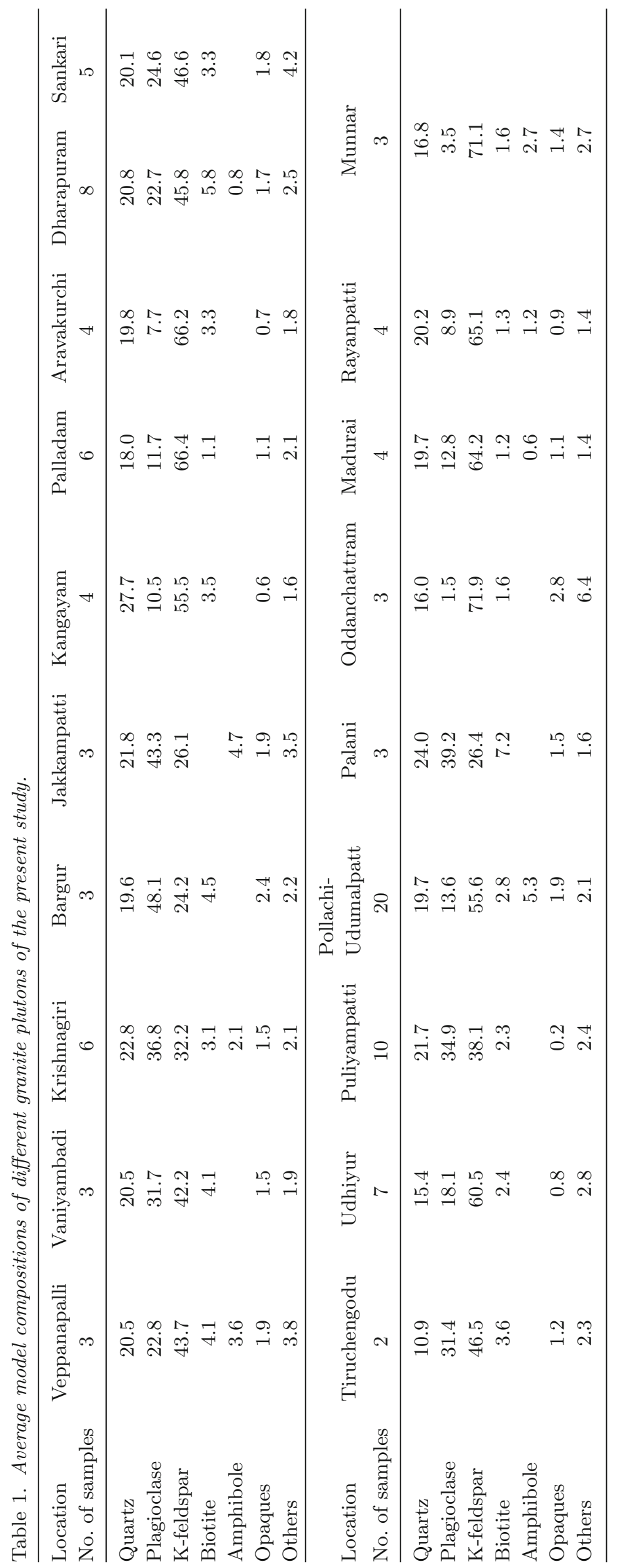



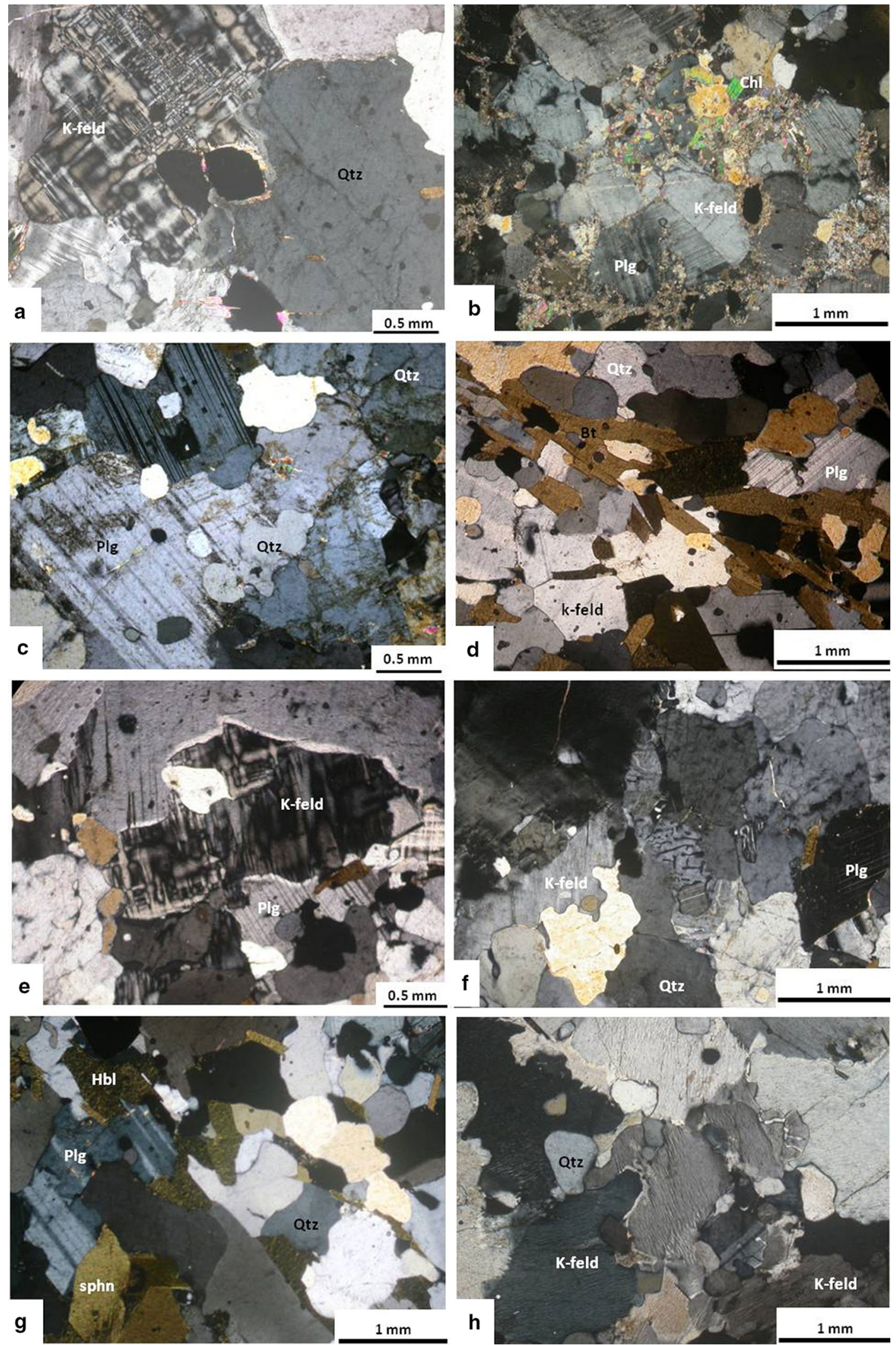

Figure 2. Microphotographs of the granitic rocks from the different blocks of southern granulite terrain showing: (a) microcline perthite in Vaniyambadi granite, (b) presence of secondary sphene, epidote, chlorite and biotite within ground mass of Vaniyambadi granites, (c) presence of inclusions and recrystallized quartz in plagioclases of Krishnagiri granite, (d) predominace of biotite occurrence in Dharapuram granite, (e) thin rims of clear albite occur around the microcline Sankari granite, (f) myrmekitic intergrowth texture in Puliyampatti granite, (g) presence of hornblende, secondary chlorite, sphene in Pollachi-Udumalpet granite, and (h) presence of orthoclase perthite as string type in Madurai granite. 


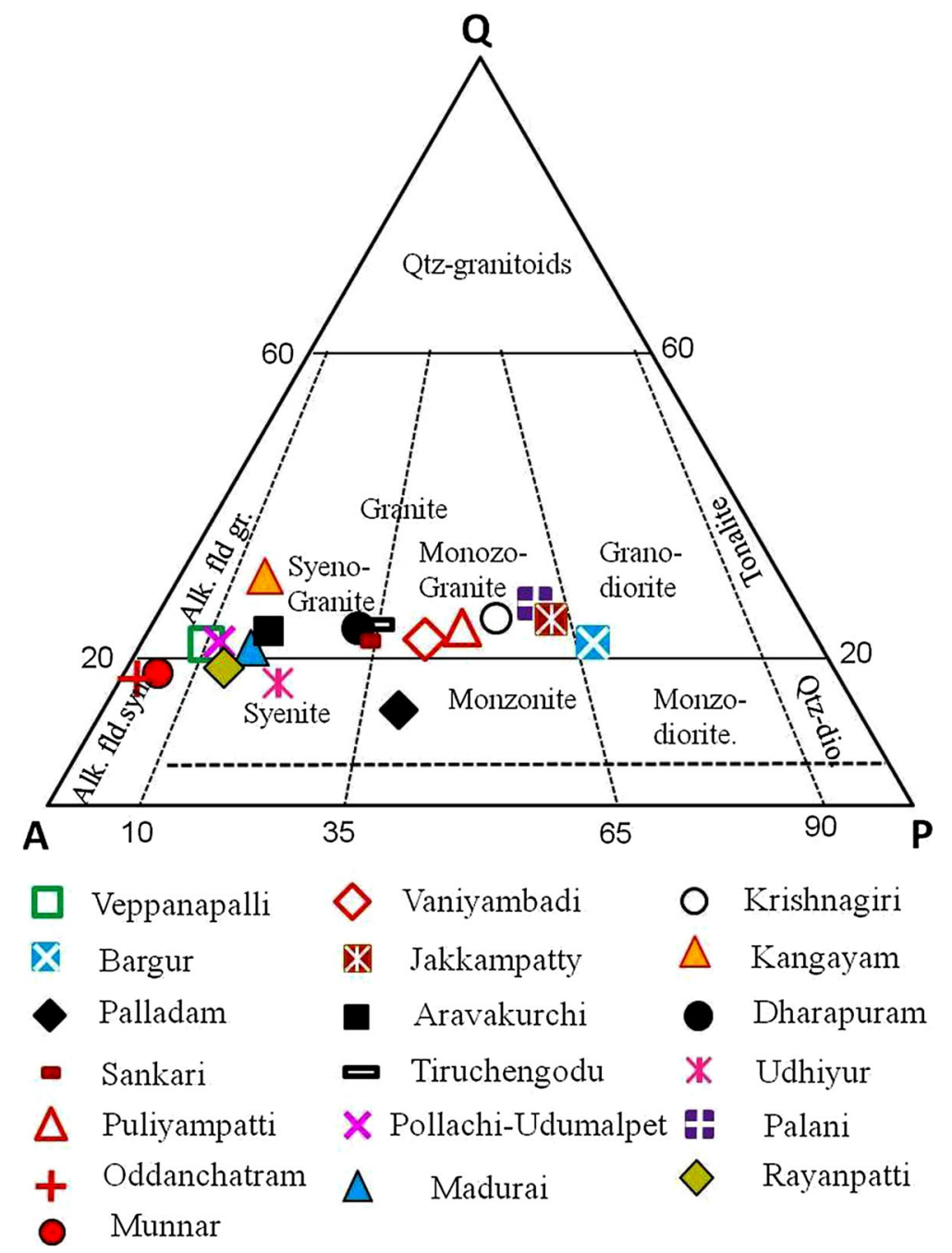

Figure 3. AFM diagram of different granitoids from the SGT.

\section{Geochemistry}

A total of 167 samples from 19 different granite plutons from the three tectonic blocks (figure 1) have been selected for major, trace and rare earth element studies. The major oxide determinations were done on a Philips Magi X PRO model PW2440, wave length dispersive X-ray fluorescence spectrometer with suitable software SUPER Q 3.0 used at NGRI laboratory and about 50 samples were analyzed at Wadia Institute of Himalayan Geology, Dehradun. The accuracy and precision of the NGRI data were given in Krishna et al. (2007). The trace elements and rare earth elements (REE) were determined by Inductively Coupled Plasma Mass Spectrometry (ICP-MS) at NGRI, Hyderabad. The samples were prepared in open acid digestion method 7:3:1 $\left(\mathrm{HF}, \mathrm{HNO}_{3}, \mathrm{HClO}_{4}\right)$ and for overall accuracy and other details see Roy et al. (2007). The ferrous iron was determined by titration method. The geochemical dataset of ranges and averages of major, trace and rare earth elements of each granite pluton was given in table 2. A careful inspection and plots of chemical compositions of various granites indicate that the major and trace elemental contents of different granite plutons vary considerably within the same granite pluton and also vary between granites across the major three tectonic blocks, namely the Northern Block, CSZ and Madurai Blocks.

The granites of Northern Block namely Veppanapalli, Vaniyambadi, Krishnagiri, Jakkampatty and Bargur are poor in $\mathrm{SiO}_{2}$ (66.9-70.4 wt.\%), $\mathrm{K}_{2} \mathrm{O}$ (0.8-4.1 wt.\%) and relatively more in $\mathrm{Al}_{2} \mathrm{O}_{3}$ (15.5-16.8 wt.\%), $\mathrm{CaO}(1.9-3.7 \%), \mathrm{MgO}$ (0.4-0.9 wt.\%) and $\mathrm{TiO}_{2}(0.2-0.4$ wt.\%) than the granites of CSZ and Madurai Block (table 2). In the CSZ, Sankari and Tiruchengodu granites show less $\mathrm{SiO}_{2}$ (64.2-74.4 wt.\%), $\mathrm{Na}_{2} \mathrm{O}$ (3-6.4 wt.\%) and $\mathrm{K}_{2} \mathrm{O}$ (1.3-5.2 wt.\%) compared to the Kangayam, 
Table 2. Average concentrations of whole rock geochemistry of different granite plutons of the present study.

\begin{tabular}{|c|c|c|c|c|c|c|c|c|c|c|}
\hline \multirow{2}{*}{$\begin{array}{l}\text { Location } \\
\text { Avg. no } \\
\mathrm{SiO}_{2}\end{array}$} & \multicolumn{2}{|c|}{$\begin{array}{l}\text { Veppanapalli } \\
\operatorname{Avg}(\mathbf{3})\end{array}$} & \multicolumn{2}{|c|}{$\begin{array}{c}\text { Vaniyambadi } \\
\operatorname{Avg}(\mathbf{2})\end{array}$} & \multicolumn{2}{|c|}{$\begin{array}{c}\text { Krishnagiri } \\
\operatorname{Avg(13)}\end{array}$} & \multicolumn{2}{|l|}{$\begin{array}{l}\text { Bargur } \\
\operatorname{Avg}(2)\end{array}$} & \multicolumn{2}{|c|}{$\begin{array}{c}\text { Jakkampatty } \\
\operatorname{Avg}(\mathbf{2})\end{array}$} \\
\hline & $66.67-67.12$ & 66.93 & $67.52-69.54$ & 68.53 & $66.71-73.98$ & 70.37 & $67.29-71.28$ & 69.29 & $61.21-66.24$ & 63.73 \\
\hline $\mathbf{T i O}_{2}$ & $0.37-0.4$ & 0.38 & $0.18-0.37$ & 0.28 & $0.1-0.58$ & 0.19 & $0.14-0.33$ & 0.24 & $0.38-0.59$ & 0.49 \\
\hline $\mathrm{Al}_{2} \mathrm{O}_{3}$ & $16.54-16.92$ & 16.79 & $16.13-16.39$ & 16.26 & $14.7-16.81$ & 16.01 & $15.18-15.89$ & 15.54 & $15.4-15.57$ & 15.49 \\
\hline $\mathbf{F e}_{2} \mathrm{O}_{3}$ & $3.02-3.22$ & $3.15^{*}$ & $1.64-1.73$ & 1.69 & $0.07-3.74$ & 1.60 & $2.72-4.11$ & $3.42^{*}$ & $3.65-4.97$ & 4.31 \\
\hline $\mathrm{FeO}$ & & & $1.12-1.12$ & 1.12 & $0.2-0.28$ & 0.22 & & & $1.56-1.92$ & 1.74 \\
\hline $\mathrm{MnO}$ & $0.04-0.05$ & 0.05 & $0.02-0.04$ & 0.03 & $0.01-0.05$ & 0.03 & $0.01-0.04$ & 0.03 & $0.04-0.07$ & 0.06 \\
\hline $\mathrm{MgO}$ & $0.83-0.94$ & 0.88 & $0.49-0.95$ & 0.72 & $0.01-1.04$ & 0.41 & $0.45-0.78$ & 0.62 & $1.49-2.48$ & 1.99 \\
\hline $\mathrm{CaO}$ & $3.59-3.75$ & 3.67 & $2.39-3.11$ & 2.75 & $0.25-3.61$ & 1.87 & $2.22-3.26$ & 2.74 & $4.1-5.8$ & 4.95 \\
\hline $\mathrm{Na}_{2} \mathrm{O}$ & $5.48-5.82$ & 5.66 & $4.51-5.11$ & 4.81 & $3.45-6.26$ & 4.81 & $3.7-4.73$ & 4.22 & $4.42-4.51$ & 4.47 \\
\hline $\mathbf{K}_{2} \mathbf{O}$ & $1.78-2.07$ & 1.92 & $3.42-3.66$ & 3.54 & $1.52-7.8$ & 4.08 & $1.44-3.87$ & 2.66 & $0.78-0.84$ & 0.81 \\
\hline $\mathbf{P}_{2} \mathbf{O}_{5}$ & $0.15-0.17$ & 0.16 & $0.06-0.21$ & 0.14 & $0.01-0.3$ & 0.09 & $0.08-0.13$ & 0.11 & $0.18-0.53$ & 0.36 \\
\hline Total & \multicolumn{2}{|c|}{99.58} & \multicolumn{2}{|c|}{99.3} & \multicolumn{2}{|c|}{99.54} & \multicolumn{2}{|c|}{99.83} & \multicolumn{2}{|c|}{98.37} \\
\hline \multicolumn{11}{|c|}{ Trace elements in ppm } \\
\hline $\mathrm{Sc}$ & $2.1-5$ & 4 & $1.1-1.2$ & 1 & $0.34-5$ & 2 & $2.01-2.4$ & 2 & $4.1-7.9$ & 6 \\
\hline $\mathbf{V}$ & $5.1-42$ & 24 & $29-29$ & 29 & $1.4-38$ & 16 & $14.2-34.9$ & 25 & $53-74$ & 64 \\
\hline $\mathrm{Cr}$ & $2.1-25$ & 17 & $25-25$ & 25 & $12-158$ & 60 & $111.6-124$ & 118 & $110-148$ & 129 \\
\hline Co & $4.1-66$ & 44 & $41-47$ & 44 & $0.6-72$ & 23 & $5.5-8.1$ & 7 & $11.4-15.5$ & 13 \\
\hline $\mathrm{Ni}$ & $2.1-11$ & 8 & $8.1-10$ & 9 & $1.9-12$ & 6 & $9.1-11$ & 10 & $14.2-27.6$ & 21 \\
\hline $\mathrm{Cu}$ & $2.1-3$ & 3 & $3.1-3.2$ & 3 & $0.3-2.4$ & 1 & $1.6-3.6$ & 3 & $3.8-4.6$ & 4 \\
\hline $\mathrm{Zn}$ & $14-64$ & 39 & $26-26$ & 26 & $5.3-73$ & 27 & $32 .-52$ & 43 & $57.2-90$ & 74 \\
\hline Ga & $8.1-25$ & 17 & $24-24$ & 24 & $10.4-25$ & 15 & $16-19$ & 17 & $20.3-21.2$ & 21 \\
\hline $\mathbf{R b}$ & $50-273$ & 134 & $64-70$ & 67 & $12-115$ & 70 & $52-84$ & 69 & $2.6-4$ & 3 \\
\hline $\mathrm{Sr}$ & $24-496$ & 303 & $328-350$ & 339 & $228-610$ & 379 & $466-495$ & 480 & $797-947$ & 872 \\
\hline $\mathbf{Y}$ & $12-122$ & 50 & $2.0-8.1$ & 5 & $1.1-18$ & 6 & $5.2-7$ & 6 & $12.1-29$ & 21 \\
\hline $\mathrm{Zr}$ & $126-249$ & 180 & 101-119 & 110 & $13-238$ & 84 & $105-106$ & 105 & 33-109 & 71 \\
\hline $\mathrm{Nb}$ & $4.7-15.7$ & 9 & $2.1-2.6$ & 2 & $0.2-7.5$ & 2 & $2.5-3.3$ & 3 & $1.8-3.8$ & 3 \\
\hline Cs & $1.2-7.1$ & 4 & $0.3-0.3$ & 0.3 & $0.04-2.8$ & 1 & $1.1-1.5$ & 1 & $0.1-0.2$ & 0.1 \\
\hline $\mathrm{Ba}$ & $464-760$ & 652 & $716-820$ & 768 & $210-3158$ & 913 & $303-1274$ & 789 & $527-634$ & 581 \\
\hline Hf & $3.2-10$ & 6 & $2.2-2.8$ & 3 & $0.4-5.4$ & 2 & $2.4-2.5$ & 2 & $1.2-2.1$ & 2 \\
\hline Ta & $0.7-2.2$ & 1.3 & $0.9-1.1$ & 1 & $0.02-1.2$ & 0.3 & $0.14-0.22$ & 0.2 & $0.1-0.4$ & 0.1 \\
\hline $\mathrm{Pb}$ & $13-32$ & 22 & $22-22$ & 22 & $16-103$ & 49 & $18-26$ & 22 & $8.3-11.4$ & 10 \\
\hline Th & n.d & & n.d & & $0.2-5.6$ & 1 & $0.8-2.3$ & 1.6 & $0.3-1.5$ & 0.9 \\
\hline $\mathbf{U}$ & n.d & & n.d & & $0.6-0.8$ & 0.3 & $0.3-0.3$ & 0.3 & $0.1-0.2$ & 0.2 \\
\hline \multicolumn{11}{|c|}{ REE in ppm } \\
\hline $\mathbf{L a}$ & $18.10-20.70$ & 19.40 & $5.05-5.05$ & 5.05 & $0.82-61.28$ & 15.53 & $4.36-10.61$ & 7.49 & $23.51-55.93$ & 39.72 \\
\hline $\mathrm{Ce}$ & $44.63-44.77$ & 44.70 & $9.73-9.73$ & 9.73 & $1.36-97.11$ & 28.02 & $8.98-22.68$ & 15.83 & $55.63-145.7$ & 99.67 \\
\hline $\mathrm{Pr}$ & $6.43-7.18$ & 6.81 & $1.17-1.17$ & 1.17 & $0.15-11.91$ & 3.50 & $1.2-2.81$ & 2.00 & $7.77-21.8$ & 14.82 \\
\hline $\mathbf{N d}$ & $22.09-26.16$ & 24.13 & $3.97-3.97$ & 3.97 & $0.52-31.63$ & 10.49 & $4.28-8.77$ & 6.53 & $26.3-78.4$ & 52.36 \\
\hline $\mathrm{Sm}$ & $3.77-8.18$ & 5.98 & $0.78-0.78$ & 0.78 & $0.51-4.64$ & 2.07 & $1.26-1.64$ & 1.45 & $5.03-12.9$ & 8.97 \\
\hline Eu & 0.09-1.02 & 0.56 & $0.82-0.82$ & 0.82 & $0.2-1.12$ & 0.75 & $0.68-0.86$ & 0.77 & $1.63-2.57$ & 2.10 \\
\hline Gd & $2.91-6.92$ & 4.92 & $0.67-0.67$ & 0.67 & $0.1-3.83$ & 1.34 & $0.68-1.21$ & 0.95 & $4.59-12.24$ & 8.41 \\
\hline $\mathrm{Tb}$ & $0.48-1.93$ & 1.21 & $0.08-0.08$ & 0.08 & $0.02-0.52$ & 0.19 & $0.12-0.21$ & 0.17 & $0.61-1.62$ & 1.11 \\
\hline Dy & $2.07-12.43$ & 7.25 & $0.35-0.35$ & 0.35 & $0.09-2.33$ & 0.85 & $0.6-0.9$ & 0.79 & $2.3-5.62$ & 3.96 \\
\hline Ho & $0.36-2.56$ & 1.46 & $0.07-0.07$ & 0.07 & $0.02-0.39$ & 0.15 & $0.11-0.18$ & 0.14 & $0.33-0.73$ & 0.53 \\
\hline Er & $1.15-8.42$ & 4.79 & $0.2-0.2$ & 0.20 & $0.09-1.39$ & 0.59 & $0.31-0.52$ & 0.42 & $1.26-2.9$ & 2.08 \\
\hline $\operatorname{Tm}$ & $0.18-1.53$ & 0.86 & $0.03-0.03$ & 0.03 & $0.02-0.3$ & 0.10 & $0.05-0.08$ & 0.06 & $0.13-0.26$ & 0.20 \\
\hline $\mathbf{Y b}$ & $1.08-1.19$ & 1.14 & $0.26-0.26$ & 0.26 & $0.12-2.48$ & 0.76 & $0.31-0.53$ & 0.42 & $1.01-2.01$ & 1.56 \\
\hline Lu & $0.22-2.03$ & 1.13 & $0.05-0.05$ & 0.05 & $0.03-0.47$ & 0.15 & $0.05-0.08$ & 0.07 & $0.15-0.31$ & 0.23 \\
\hline$\Sigma$ REE & $107.1-141.5$ & 124.3 & $23.23-23.23$ & 23.23 & $6.4-213.82$ & 64.51 & $23.16-50.98$ & 37.1 & $128.3-343.2$ & 235.7 \\
\hline
\end{tabular}


Table 2. (Continued.)

\begin{tabular}{|c|c|c|c|c|c|c|c|c|c|c|}
\hline \multirow{2}{*}{$\begin{array}{l}\text { Location } \\
\text { Avg. no } \\
\mathrm{SiO}_{2}\end{array}$} & \multicolumn{2}{|c|}{$\begin{array}{c}\text { Kangayam } \\
\mathbf{A v g}(\mathbf{8})\end{array}$} & \multicolumn{2}{|l|}{$\begin{array}{c}\text { Palladam } \\
\operatorname{Avg}(\mathbf{8})\end{array}$} & \multicolumn{2}{|c|}{$\begin{array}{c}\text { Aravakurchi } \\
\operatorname{Avg}(\mathbf{4})\end{array}$} & \multicolumn{2}{|c|}{$\begin{array}{c}\text { Dharapuram } \\
\operatorname{Avg}(\mathbf{7})\end{array}$} & \multicolumn{2}{|l|}{$\begin{array}{l}\text { Sankari } \\
\operatorname{Avg}(5)\end{array}$} \\
\hline & $65.05-74.06$ & 71.54 & $70.13-73.88$ & 71.92 & $72.38-76.16$ & 73.36 & $58.78-74.17$ & 69.06 & $69.8-74.46$ & 72.67 \\
\hline $\mathrm{TiO}_{2}$ & $0.01-0.14$ & 0.07 & $0.01-0.28$ & 0.09 & $0.03-0.12$ & 0.07 & $0.01-0.79$ & 0.21 & $0.04-0.08$ & 0.06 \\
\hline $\mathrm{Al}_{2} \mathrm{O}_{3}$ & $14.54-18.24$ & 15.81 & $14.43-15.87$ & 15.17 & $14.29-15.2$ & 14.85 & $14.94-18.24$ & 15.69 & $14.9-16.59$ & 15.38 \\
\hline $\mathrm{Fe}_{2} \mathrm{O}_{3}$ & $0.3-2.27$ & 0.91 & $0.13-2.04$ & 1.07 & $0.92-1.11$ & 1.01 & $0.72-5.75$ & 2.03 & $0.71-1.71$ & 1.16 \\
\hline $\mathrm{FeO}$ & $0.52-0.92$ & 0.75 & $0.2-0.28$ & 0.24 & $0.24-0.48$ & 0.36 & $0.48-3.64$ & 1.43 & $0.40-0.60$ & 0.48 \\
\hline $\mathrm{MnO}$ & $0.01-0.04$ & 0.02 & $0.01-0.03$ & 0.01 & $0.01-0.01$ & 0.01 & $0.01-0.09$ & 0.02 & $0.01-0.02$ & 0.01 \\
\hline $\mathrm{MgO}$ & $0.04-0.6$ & 0.18 & $0.01-0.43$ & 0.13 & $0.1-0.2$ & 0.16 & $0.1-0.97$ & 0.43 & $0.05-0.20$ & 0.10 \\
\hline $\mathrm{CaO}$ & $1.04-3.78$ & 1.54 & $0.37-2.22$ & 1.16 & $1.02-1.53$ & 1.18 & $1.83-3.86$ & 2.59 & $0.67-1.28$ & 1.02 \\
\hline $\mathrm{Na}_{2} \mathrm{O}$ & $3.03-5.34$ & 4.39 & $1.97-6.32$ & 4.02 & $3.04-4.08$ & 3.71 & $3.11-5.34$ & 4.27 & $3.83-6.4$ & 4.46 \\
\hline $\mathbf{K}_{2} \mathbf{O}$ & $2.73-6.17$ & 4.68 & $3.31-5.96$ & 5.08 & $2.96-6.05$ & 4.97 & $2.73-6.03$ & 4.13 & $1.62-5.17$ & 4.32 \\
\hline $\mathbf{P}_{2} \mathbf{O}_{5}$ & $0.01-0.1$ & 0.03 & $0.01-0.11$ & 0.03 & $0.02-0.02$ & 0.02 & $0.02-0.37$ & 0.11 & $0.01-0.03$ & 0.02 \\
\hline Total & \multicolumn{2}{|c|}{99.43} & \multicolumn{2}{|c|}{98.8} & \multicolumn{2}{|c|}{99.5} & \multicolumn{2}{|c|}{99.34} & \multicolumn{2}{|c|}{99.49} \\
\hline \multicolumn{11}{|c|}{ Trace elements in ppm } \\
\hline Sc & $1.2-6.4$ & 2 & $0.4-3.1$ & 2 & $0.7-3.8$ & 2 & $1.8-12.3$ & 5 & $2.5-3.2$ & 3 \\
\hline $\mathbf{V}$ & $3.2-31$ & 13 & $1.3-16.2$ & 7 & $1.9-6.7$ & 4 & $2.3-63.6$ & 22 & $6.5-27.3$ & 11 \\
\hline $\mathrm{Cr}$ & $28-119$ & 75 & $8.2-137.9$ & 68 & $32-204$ & 96 & $14.7-168.9$ & 78 & $59-138.5$ & 104 \\
\hline Co & $0.78-73$ & 26 & $08-63.1$ & 11 & $1.5-2.3$ & 2 & $1.5-14.3$ & 5 & $1.03-3.1$ & 2 \\
\hline $\mathrm{Ni}$ & $3.7-25$ & 10 & $2.1-5.1$ & 3 & $2.3-7.2$ & 4 & $2.9-9.4$ & 7 & $0.8-1.5$ & 5 \\
\hline $\mathrm{Cu}$ & $0.9-3.1$ & 2 & $1.2-9.4$ & 3 & $1.1-1.9$ & 2 & $0.3-3.5$ & 2 & $20.8-48.3$ & 1 \\
\hline $\mathrm{Zn}$ & $11.1-42.2$ & 27 & $13.1-25$ & 15 & $12.9-24.5$ & 17 & $9.7-91.3$ & 38 & $16.5-24.9$ & 32 \\
\hline $\mathrm{Ga}$ & $11.2-26.3$ & 18 & $23-97$ & 17 & $14.1-24.9$ & 20 & $16.2-27.5$ & 21 & $64.4-155-9$ & 21 \\
\hline $\mathbf{R b}$ & $26-111$ & 81 & $23.1-97$ & 70 & $22-120$ & 86 & 26-129 & 71 & $64-156$ & 116 \\
\hline $\mathrm{Sr}$ & $101-893$ & 392 & $113-869$ & 440 & $196-304$ & 238 & $311-1152$ & 653 & $118-892$ & 284 \\
\hline $\mathbf{Y}$ & $3.4-19$ & 10 & $1.2-20$ & 6 & $0.5-5.4$ & 3 & $0.9-145$ & 29 & $3.9-18.7$ & 8 \\
\hline $\mathrm{Zr}$ & $18-140$ & 73 & $10-471$ & 127 & $24-217$ & 136 & $16-512$ & 108 & $33-269$ & 163 \\
\hline $\mathrm{Nb}$ & $0.23-6.4$ & 2 & $0.07-12.2$ & 3 & $0.11-5.2$ & 3 & $0.8-25.1$ & 6 & $2.5-10.9$ & 5 \\
\hline Cs & $0.03-0.47$ & 0.2 & $0.01-0.1$ & 0.1 & $0.08-0.16$ & 0.1 & $0.03-0.2$ & 0.1 & $0.26-0.7$ & 1 \\
\hline $\mathrm{Ba}$ & $193-1655$ & 851 & $748-6864$ & 3298 & $822-2971$ & 1962 & $1188-9187$ & 3145 & $305-1271$ & 577 \\
\hline Hf & $0.6-3.5$ & 2 & $0.43-11.1$ & 3 & $0.64-7.7$ & 4 & $0.52-12.5$ & 3 & $0.99-8.8$ & 6 \\
\hline $\mathbf{T a}$ & $0.01-6.2$ & 1.1 & $0.01-1.4$ & 0.3 & $0.01-0.09$ & 0.03 & $0.06-1.3$ & 0.3 & $0.05-1.05$ & 0.34 \\
\hline $\mathrm{Pb}$ & $34.1-55$ & 42 & $22.3-55$ & 36 & $17.4-92.1$ & 66 & $22.9-44.1$ & 34 & $22.7-82.9$ & 68 \\
\hline Th & $0.2-7.3$ & 3 & $0.1-33$ & 7 & $0.18-66.8$ & 38 & $0.2-17.7$ & 5.6 & $0.29-34.9$ & 18 \\
\hline $\mathbf{U}$ & $0.2-0.5$ & 0.3 & $0.03-3.2$ & 0.6 & $0.1-4.2$ & 1.8 & $0.12-1.37$ & 0.3 & $0.4-4.13$ & 2.4 \\
\hline \multicolumn{11}{|c|}{ REE in ppm } \\
\hline La & $2.68-26.31$ & 12.28 & $1.22-48.94$ & 17.93 & $9.77-46.21$ & 29.63 & $8.99-82.45$ & 33.65 & $2.03-8.36$ & 4.90 \\
\hline $\mathrm{Ce}$ & $4.71-45.65$ & 22.65 & $2.38-91.94$ & 33.23 & $18.8-106.5$ & 65.95 & $17.17-217.5$ & 72.34 & $4.84-21.2$ & 11.91 \\
\hline $\operatorname{Pr}$ & $0.57-5.74$ & 2.70 & $0.27-9.79$ & 3.39 & $2.01-9.88$ & 6.49 & $1.41-33.36$ & 9.58 & $0.74-3.08$ & 1.79 \\
\hline $\mathrm{Nd}$ & $1.78-18.14$ & 8.13 & $0.76-27.1$ & 10.44 & $4.96-33.68$ & 20.96 & $4.19-119.38$ & 32.41 & $2.74-10.89$ & 6.42 \\
\hline $\mathrm{Sm}$ & $0.52-4.52$ & 2.06 & $1.07-5.73$ & 3.19 & $1.71-5.23$ & 3.65 & $1.33-26.25$ & 7.25 & $0.9-3.44$ & 2.20 \\
\hline Eu & $0.37-1.24$ & 0.97 & $0.62-2.97$ & 1.27 & $0.97-1.17$ & 1.04 & $1.09-3.34$ & 1.82 & $0.39-0.68$ & 0.58 \\
\hline Gd & $0.39-3.43$ & 1.61 & $0.08-4.27$ & 1.44 & $1.02-3.03$ & 2.40 & $0.42-19.77$ & 5.48 & $0.52-2.21$ & 1.37 \\
\hline $\mathrm{Tb}$ & $0.07-0.43$ & 0.22 & $0.01-0.65$ & 0.19 & $0.06-0.34$ & 0.23 & $0.04-4.09$ & 0.91 & $0.12-0.35$ & 0.25 \\
\hline Dy & $0.45-1.93$ & 1.18 & $0.07-2.82$ & 0.90 & $0.14-1.45$ & 0.80 & $0.18-21.31$ & 4.47 & $0.58-1.63$ & 1.13 \\
\hline Ho & $0.09-0.43$ & 0.23 & $0.01-0.49$ & 0.13 & $0.01-0.11$ & 0.06 & $0.02-3.87$ & 0.77 & $0.1-0.4$ & 0.19 \\
\hline Er & $0.38-1.58$ & 0.89 & $0.06-1.51$ & 0.46 & $0.09-0.37$ & 0.25 & $0.05-11.59$ & 2.41 & $0.30-1.46$ & 0.58 \\
\hline $\mathbf{T m}$ & $0.05-0.3$ & 0.15 & $0.01-0.21$ & 0.07 & $0.01-0.02$ & 0.02 & $0.01-1.81$ & 0.35 & $0.04-0.29$ & 0.10 \\
\hline $\mathbf{Y b}$ & $0.4-2.35$ & 1.19 & $0.06-1.64$ & 0.70 & $0.04-0.22$ & 0.14 & $0.07-11.78$ & 2.37 & $0.28-2.28$ & 0.75 \\
\hline Lu & $0.06-0.41$ & 0.21 & $0.01-0.34$ & 0.15 & $0.01-0.04$ & 0.02 & $0.01-1.82$ & 0.37 & $0.05-0.38$ & 0.13 \\
\hline$\Sigma$ REE & $14.58-106.6$ & 54.45 & $10.01-184.01$ & 73.5 & $39.8-208.1$ & 131.64 & $35.7-558.2$ & 174.2 & $18.41-52.62$ & 32.3 \\
\hline
\end{tabular}


Table 2. (Continued.)

\begin{tabular}{|c|c|c|c|c|c|c|c|c|c|c|}
\hline \multirow{2}{*}{$\begin{array}{l}\text { Location } \\
\text { Avg. no } \\
\mathrm{SiO}_{2}\end{array}$} & \multicolumn{2}{|c|}{$\begin{array}{l}\text { Tiruchengodu } \\
\mathbf{A v g}(\mathbf{6})\end{array}$} & \multicolumn{2}{|c|}{$\begin{array}{l}\text { Udhiyur } \\
\operatorname{Avg}(11)\end{array}$} & \multicolumn{2}{|c|}{$\begin{array}{l}\text { Puliyampatti } \\
\operatorname{Avg}(\mathbf{1 6})\end{array}$} & \multicolumn{2}{|c|}{$\begin{array}{l}\text { Pollachi-Udumalpet } \\
\qquad \operatorname{Avg}(\mathbf{3 3})\end{array}$} & \multicolumn{2}{|c|}{$\begin{array}{c}\text { Palani } \\
\operatorname{Avg}(\mathbf{1 0})\end{array}$} \\
\hline & $48.43-72.61$ & 64.2 & $71.4-73.82$ & 72.3 & $68.17-73.59$ & 71.79 & $62.89-76.19$ & 70.36 & $63.15-72.07$ & 68.02 \\
\hline $\mathrm{TiO}_{2}$ & $0.19-1.65$ & 0.66 & $0.01-0.08$ & 0.03 & $0.01-0.25$ & 0.06 & $0.01-0.85$ & 0.29 & $0.1-0.47$ & 0.33 \\
\hline $\mathrm{Al}_{2} \mathrm{O}_{3}$ & $13.72-17.94$ & 15.03 & $14.36-16.51$ & 15.36 & $15.07-17.15$ & 15.85 & $13.13-19.96$ & 14.87 & $14.87-16.78$ & 15.97 \\
\hline $\mathbf{F e}_{2} \mathrm{O}_{3}$ & $2.4-14.74$ & 5.81 & $0.15-0.82$ & 0.44 & $0.03-2.33$ & 0.42 & $0.25-4.55$ & 1.81 & $0.91-4.35$ & 2.48 \\
\hline $\mathrm{FeO}$ & $3.28-3.76$ & 3.58 & $0.12-0.28$ & 0.15 & $0.20-0.80$ & 0.32 & $0.2-2.08$ & 0.71 & $0.68-3.1$ & 1.45 \\
\hline $\mathrm{MnO}$ & $0.01-0.1$ & 0.05 & $0.01-0.08$ & 0.03 & $0.01-0.04$ & 0.01 & $0.01-0.09$ & 0.02 & $0.01-0.06$ & 0.03 \\
\hline $\mathrm{MgO}$ & $0.39-8.38$ & 2.11 & $0.01-0.15$ & 0.06 & $0.01-0.89$ & 0.14 & $0.01-1.18$ & 0.41 & $0.26-1.61$ & 0.83 \\
\hline $\mathrm{CaO}$ & $1.46-4.43$ & 2.50 & $1.04-1.69$ & 1.28 & $1.03-3.05$ & 2.17 & $0.52-4.69$ & 1.68 & $1.44-5.54$ & 3.32 \\
\hline $\mathrm{Na}_{2} \mathrm{O}$ & $3.01-4.94$ & 3.12 & $3.55-6.42$ & 5.18 & $3.91-8.12$ & 6.5 & $2.62-6.26$ & 3.85 & $3.03-5.48$ & 4.42 \\
\hline $\mathbf{K}_{2} \mathbf{O}$ & $1.29-4.56$ & 2.26 & $2.61-5.18$ & 4.03 & $0.5-5.18$ & 1.86 & $1.52-6.93$ & 5.12 & $1.18-6.28$ & 2.83 \\
\hline $\mathbf{P}_{2} \mathbf{O}_{5}$ & $0.04-0.52$ & 0.15 & $0.01-0.04$ & 0.01 & $0.01-0.12$ & 0.03 & $0.01-0.5$ & 0.14 & $0.01-0.28$ & 0.15 \\
\hline Total & \multicolumn{2}{|c|}{85.65} & \multicolumn{2}{|c|}{98.8} & \multicolumn{2}{|c|}{99.16} & \multicolumn{2}{|c|}{99.11} & \multicolumn{2}{|c|}{99.23} \\
\hline \multicolumn{11}{|c|}{ Trace elements in ppm } \\
\hline Sc & $2.9-21$ & 7 & $1.2-2.25$ & 1 & $0.4-21$ & 3 & $0.7-12$ & 3 & $2.3-8$ & 4 \\
\hline $\mathbf{V}$ & $24.5-182$ & 62 & $0.96-31$ & 8 & $4.2-14$ & 8 & $2.3-41$ & 20 & $20.5-57$ & 36 \\
\hline $\mathrm{Cr}$ & $92.8-301$ & 149 & $4.1-128$ & 36 & $9.1-161$ & 104 & $8.1-158$ & 94 & $10.7-139$ & 81 \\
\hline Co & $2.9-49$ & 16 & $0.72-75$ & 25 & $1.3-63$ & 12 & $1.2-64$ & 10 & $2.3-71$ & 23 \\
\hline $\mathrm{Ni}$ & $6.9-92$ & 29 & $2.2-25$ & 5 & $2.2-5$ & 3 & $2.3-12$ & 5 & $3.3-12.5$ & 7 \\
\hline $\mathrm{Cu}$ & $1.5-8.6$ & 4 & $0.31-3.2$ & 2 & $1.3-4$ & 2 & $0.4-9$ & 3 & $0.76-6$ & 3 \\
\hline $\mathrm{Zn}$ & $30.9-124$ & 58 & $4.5-17$ & 10 & $8.1-59$ & 17 & $9.2-84$ & 41 & 22.9-69 & 47 \\
\hline $\mathrm{Ga}$ & $14.5-36$ & 18 & $30.1-86$ & 18 & $12.1-21$ & 16 & $12.2-50$ & 21 & $15.6-24$ & 20 \\
\hline $\mathbf{R b}$ & $7.34-143$ & 57 & $30-86$ & 55 & $6.1-85$ & 27 & $16-151$ & 94 & 16-104 & 47 \\
\hline $\mathrm{Sr}$ & $248-456$ & 285 & $128-1026$ & 416 & $217-1205$ & 679 & $66-1955$ & 662 & $461-1187$ & 697 \\
\hline $\mathbf{Y}$ & $6.1-96$ & 25 & $1.2-13$ & 4 & $0.6-4$ & 2 & $1.2-91$ & 19 & $4.2-23$ & 10 \\
\hline $\mathrm{Zr}$ & $16-130$ & 76 & $2-109$ & 28 & $37-322$ & 115 & $11-285$ & 118 & $12-106$ & 46 \\
\hline $\mathrm{Nb}$ & $2.1-47.5$ & 11 & $0.17-1.5$ & 1 & $0.4-8$ & 2 & $0.18-26$ & 8 & $1.6-11$ & 4 \\
\hline Cs & $0.02-1.8$ & 0.4 & $0.03-0.22$ & 0.1 & $0.01-0.3$ & 0.1 & $0.02-0.23$ & 0.1 & $0.02-0.22$ & 0.1 \\
\hline $\mathrm{Ba}$ & $478-1777$ & 746 & $211-4286$ & 1865 & $6.8-1821$ & 447 & 295-8909 & 2301 & 359-3210 & 1102 \\
\hline Hf & $0.39-3.1$ & 2 & $0.08-3.5$ & 1 & $0.7-9$ & 3 & $0.2-9$ & 3 & $0.34-3.2$ & 1 \\
\hline Ta & $0.03-2.9$ & 1 & $0.03-1.1$ & 0.3 & $0.01-0.9$ & 0.2 & $0.03-1.3$ & 0.4 & $0.01-1.3$ & 0.4 \\
\hline $\mathrm{Pb}$ & $26-66$ & 39 & $31.8-38.5$ & 36 & $4.2-389$ & 58 & $11.3-80$ & 44 & $11.0-85$ & 30 \\
\hline Th & $0.17-56.16$ & 22 & $0.13-0.24$ & 0.2 & $0.1-8.5$ & 2.4 & $0.4-124$ & 37 & $0.5-26$ & 6.6 \\
\hline $\mathbf{U}$ & $0.1-1.38$ & 0.7 & $0.02-0.1$ & 0.04 & $0.1-1.1$ & 0.4 & $0.1-6$ & 0.6 & $0.1-0.3$ & 0.2 \\
\hline \multicolumn{11}{|c|}{ REE in ppm } \\
\hline La & $6.81-139.6$ & 52.71 & $0.16-24.96$ & 3.94 & $0.06-6.02$ & 3.51 & $7.07-1171$ & 134.6 & $4.24-51.78$ & 25.88 \\
\hline $\mathrm{Ce}$ & $15.45-319.8$ & 115.2 & $0.27-36.21$ & 6.22 & $1.10-13.03$ & 7.09 & $10.3-2153$ & 356.3 & $9.98-96.21$ & 49.51 \\
\hline $\operatorname{Pr}$ & $2.14-43.2$ & 15.10 & $0.03-3.67$ & 0.67 & $0.14-1.68$ & 0.93 & $1.00-69.52$ & 22.67 & $1.44-12.92$ & 5.96 \\
\hline $\mathrm{Nd}$ & $7.81-141.5$ & 48.17 & $0.12-9.16$ & 1.89 & $0.48-6.23$ & 3.20 & $2.55-124.4$ & 46.24 & $5.41-43.51$ & 18.72 \\
\hline $\mathrm{Sm}$ & $2.09-24.2$ & 8.18 & $0.35-1.02$ & 0.62 & $0.17-2.32$ & 0.89 & $0.87-20.02$ & 8.47 & $1.75-8.94$ & 3.36 \\
\hline Eu & $0.88-3.71$ & 1.67 & $0.18-1.16$ & 0.41 & $0.26-0.88$ & 0.49 & $0.47-12.84$ & 2.23 & $0.73-2.32$ & 1.22 \\
\hline Gd & $1.54-18.18$ & 6.08 & $0.06-1.16$ & 0.32 & $0.08-1.92$ & 0.62 & $0.23-13.91$ & 6.27 & $1.09-5.55$ & 2.55 \\
\hline $\mathrm{Tb}$ & $0.24-3.18$ & 1.01 & $0.01-0.19$ & 0.05 & $0.04-0.20$ & 0.09 & $0.02-4.26$ & 0.91 & $0.21-0.90$ & 0.37 \\
\hline Dy & $1.11-15.47$ & 4.58 & $0.16-1.35$ & 0.39 & $0.07-0.63$ & 0.35 & 0.09-11.99 & 3.16 & $0.76-3.39$ & 1.60 \\
\hline Ho & $0.16-2.56$ & 0.75 & $0.02-0.26$ & 0.07 & $0.01-0.50$ & 0.08 & $0.01-2.28$ & 0.56 & $0.11-0.60$ & 0.26 \\
\hline Er & $0.49-7.48$ & 2.25 & $0.10-1.12$ & 0.31 & $0.04-0.32$ & 0.16 & $0.05-7.5$ & 1.54 & $0.43-1.72$ & 0.82 \\
\hline $\mathbf{T m}$ & $0.03-1.06$ & 0.32 & $0.02-0.20$ & 0.06 & $0.01-0.05$ & 0.02 & $0.01-1.19$ & 0.23 & $0.04-0.23$ & 0.11 \\
\hline $\mathbf{Y b}$ & $0.18-6.62$ & 2.16 & $0.16-1.55$ & 0.51 & $0.05-0.32$ & 0.16 & $0.05-8.09$ & 1.35 & $0.28-1.48$ & 0.71 \\
\hline Lu & $0.03-1.01$ & 0.36 & $0.03-0.30$ & 0.10 & $0.01-0.05$ & 0.03 & $0.01-28.0$ & 1.20 & $0.04-0.22$ & 0.12 \\
\hline$\Sigma$ REE & $47.57-727.6$ & 258.5 & $1.87-78.13$ & 15.6 & $2.6-30.79$ & 18.33 & $23.5-3432$ & 582.0 & $28.7-215.5$ & 111.2 \\
\hline
\end{tabular}


Table 2. (Continued.)

\begin{tabular}{|c|c|c|c|c|c|c|c|c|}
\hline \multirow{2}{*}{$\begin{array}{l}\text { Location } \\
\text { Avg. no } \\
\mathrm{SiO}_{2}\end{array}$} & \multicolumn{2}{|c|}{$\begin{array}{l}\text { Oddanchattram } \\
\qquad \mathbf{A v g}(\mathbf{6})\end{array}$} & \multicolumn{2}{|c|}{$\begin{array}{l}\text { Madurai } \\
\operatorname{Avg}(\mathbf{1 7})\end{array}$} & \multicolumn{2}{|c|}{$\begin{array}{c}\text { Rayanpatti } \\
\mathbf{A v g}(\mathbf{3})\end{array}$} & \multicolumn{2}{|c|}{$\begin{array}{l}\text { Munnar } \\
\operatorname{Avg(10)}\end{array}$} \\
\hline & $66.84-74.81$ & 69.31 & $68.92-74.24$ & 72.48 & $72.12-74.44$ & 73.2 & $67.64-73.36$ & 71.72 \\
\hline $\mathrm{TiO}_{2}$ & $0.15-0.78$ & 0.42 & $0.15-0.77$ & 0.36 & $0.14-0.43$ & 0.29 & $0.11-0.46$ & 0.24 \\
\hline $\mathrm{Al}_{2} \mathrm{O}_{3}$ & $1177-16.41$ & 14.84 & $11.87-13.55$ & 12.59 & $12.12-13.31$ & 12.7 & $12.02-13.31$ & 12.71 \\
\hline $\mathrm{Fe}_{2} \mathrm{O}_{3}$ & $1.39-4.2$ & 2.37 & $1.41-3.69$ & $2.71^{*}$ & $1.24-3.59$ & $2.35^{*}$ & $1.49-2.74$ & $2.37^{*}$ \\
\hline $\mathrm{FeO}$ & $0.72-1.92$ & 1.11 & & & & & & \\
\hline $\mathrm{MnO}$ & $0.01-0.07$ & 0.03 & $0.02-0.05$ & 0.03 & $0.01-0.02$ & 0.01 & $0.01-0.05$ & 0.03 \\
\hline $\mathrm{MgO}$ & $0.25-0.88$ & 0.59 & $0.12-0.93$ & 0.44 & $0.17-0.40$ & 0.26 & $0.29-1.39$ & 0.64 \\
\hline $\mathrm{CaO}$ & $1.1-4.53$ & 2.40 & $0.82-2.12$ & 1.35 & $0.82-1.9$ & 1.30 & $1.12-2.22$ & 1.62 \\
\hline $\mathrm{Na}_{2} \mathrm{O}$ & $0.96-4.87$ & 3.51 & $2.44-3.37$ & 3.06 & $1.9-2.45$ & 2.15 & $2.65-3.66$ & 3.16 \\
\hline $\mathbf{K}_{2} \mathbf{O}$ & $1.04-8.09$ & 3.90 & $4.71-5.85$ & 5.31 & $5.31-6.43$ & 5.75 & $3.79-7.06$ & 5.6 \\
\hline $\mathbf{P}_{2} \mathbf{O}_{5}$ & $0.05-0.29$ & 0.17 & $0.01-0.22$ & 0.11 & $0.01-0.10$ & 0.05 & $0.06-0.52$ & 0.17 \\
\hline Total & \multicolumn{2}{|c|}{98.66} & \multicolumn{2}{|c|}{98.4} & \multicolumn{2}{|c|}{98.12} & \multicolumn{2}{|c|}{98.3} \\
\hline \multicolumn{9}{|c|}{ Trace elements in ppm } \\
\hline Sc & $2.8-23$ & 7 & $1.6-6$ & 3 & $1.2-5$ & 3 & $0.4-6$ & 3 \\
\hline $\mathbf{V}$ & $15.3-79$ & 39 & $2.5-30$ & 9 & $4.1-11$ & 7 & $1.1-17$ & 9 \\
\hline $\mathrm{Cr}$ & $22.8-115$ & 85 & $0.3-11$ & 6 & $8.2-11$ & 10 & $0.4-8$ & 6 \\
\hline Co & $2.9-14$ & 8 & $1.2-7$ & 4 & $3.3-6$ & 5 & $0.5-8$ & 4 \\
\hline $\mathrm{Ni}$ & $7.1-26$ & 13 & $1.7-7$ & 3 & $2.2-3$ & 3 & $1.1-5$ & 3 \\
\hline $\mathrm{Cu}$ & $0.38-16$ & 4 & $0.3-2$ & 1 & $0.3-0.4$ & 0.1 & $0.1-2$ & 0.7 \\
\hline $\mathrm{Zn}$ & $30-80$ & 53 & $11.8-39$ & 24 & $13-23$ & 20 & $2.2-34$ & 19 \\
\hline Ga & $13-26$ & 20 & $1.8-25$ & 20 & $13-21$ & 17 & $2.1-24$ & 15 \\
\hline $\mathbf{R b}$ & 19-189 & 87 & $1-377$ & 230 & $228-253$ & 241 & $11-189$ & 90 \\
\hline $\mathrm{Sr}$ & $61-1129$ & 689 & $5-1554$ & 366 & $7.2-71$ & 29 & $60-795$ & 259 \\
\hline $\mathbf{Y}$ & $9.2-33$ & 21 & $1.2-62$ & 26 & $4.3-30$ & 15 & $2.3-43$ & 21 \\
\hline $\mathrm{Zr}$ & $80-3544$ & 793 & $8-269$ & 106 & $166-2366$ & 1521 & $3.4-1685$ & 232 \\
\hline $\mathrm{Nb}$ & $4.9-33$ & 16 & $4.2-30$ & 18 & $6.1-13$ & 10 & $1.3-32$ & 13 \\
\hline Cs & $0.04-3.3$ & 1 & $0.14-2.44$ & 1 & $2.3-3$ & 3 & $0.04-0.04$ & 0.2 \\
\hline $\mathbf{B a}$ & $201-4447$ & 1822 & $181-3595$ & 793 & $322-564$ & 448 & $239-2737$ & 1191 \\
\hline Hf & $2.45-71$ & 16 & $0.24-8$ & 3 & $5.1-8$ & 7 & $0.08-5$ & 2 \\
\hline $\mathrm{Ta}$ & $0.24-2.8$ & 1 & $0.2-2$ & 1 & $0.3-0.5$ & 0.4 & $0.04-2$ & 0.6 \\
\hline $\mathrm{Pb}$ & $10.1-153$ & 65 & $30-43$ & 38 & $33.8-36$ & 35 & $4.2-65$ & 37 \\
\hline Th & $4.3-57$ & 22 & $1.2-2606$ & 262 & $2.1-54$ & 30 & $16-1680$ & 228 \\
\hline $\mathbf{U}$ & $0.4-3.7$ & 1.5 & $0.16-5.6$ & 1.4 & $3.2-4.1$ & 3.3 & $0.2-2.37$ & 0.8 \\
\hline \multicolumn{9}{|c|}{ REE in ppm } \\
\hline La & $19.96-125.5$ & 54.13 & $11.09-169$ & 75.31 & $8.18-223$ & 93.45 & $39.18-259$ & 59.48 \\
\hline $\mathrm{Ce}$ & $55.3-279.4$ & 118.42 & $21.66-304$ & 196.45 & $15.72-441$ & 235.36 & $70.6-437$ & 126.45 \\
\hline Pr & $7.35-35.43$ & 14.30 & $2.16-31.23$ & 18.71 & $1.56-43.9$ & 25.24 & $9.13-63.33$ & 24.16 \\
\hline $\mathbf{N d}$ & $27.6-107.14$ & 44.34 & $8.35-107.9$ & 66.56 & $6.18-148.65$ & 87.55 & $20.23-133.3$ & 62.08 \\
\hline $\mathrm{Sm}$ & $4.42-18.94$ & 8.33 & $1.99-15.84$ & 10.62 & $1.85-17.79$ & 11.95 & $2.10-18.45$ & 9.02 \\
\hline Eu & $1.04-4.39$ & 2.14 & $0.42-2.72$ & 1.17 & $1.19-1.63$ & 1.40 & $0.31-3.28$ & 1.49 \\
\hline Gd & $3.03-19.9$ & 7.27 & $0.89-12.99$ & 8.11 & $1.38-14.02$ & 9.53 & $1.72-13.2$ & 6.81 \\
\hline $\mathbf{T b}$ & $0.43-2.03$ & 0.89 & $0.12-1.84$ & 1.10 & $0.18-1.52$ & 0.93 & $0.11-1.28$ & 0.77 \\
\hline Dy & $1.72-6.76$ & 3.77 & $0.67-11.34$ & 6.69 & $0.85-8.23$ & 4.37 & $0.42-7.64$ & 4.22 \\
\hline Ho & $0.26-0.83$ & 0.50 & $0.06-1.22$ & 0.70 & $0.07-0.73$ & 0.35 & $0.03-0.83$ & 0.42 \\
\hline Er & $0.70-3.55$ & 1.80 & $0.21-3.97$ & 2.32 & $0.17-1.97$ & 0.97 & $0.11-2.85$ & 1.39 \\
\hline $\mathbf{T m}$ & $0.09-0.31$ & 0.21 & $0.02-0.51$ & 0.29 & $0.02-0.16$ & 0.08 & $0.01-0.36$ & 0.16 \\
\hline $\mathbf{Y b}$ & $0.55-2.76$ & 1.62 & $0.16-5.14$ & 2.81 & $0.21-1.36$ & 0.69 & $0.09-3.69$ & 1.58 \\
\hline Lu & $0.10-0.45$ & 0.26 & $0.02-0.81$ & 0.44 & $0.04-0.19$ & 0.10 & $0.01-0.59$ & 0.25 \\
\hline$\Sigma$ REE & $127.7-606.8$ & 257.9 & $48.2-687$ & 391.2 & $37.6-884.5$ & 471.2 & 135.6-917.2 & 248.2 \\
\hline
\end{tabular}


Puliyampatti, Pollachi-Udumalpet granites. The Tiruchengodu granites have less silica and more iron compared to the Sankari granites. The grey and pink phases of granites are common and the pink phase contains higher $\mathrm{K}_{2} \mathrm{O}$ than the grey types because of sub-solidus potassic fluid alteration. The Kangayam-Palladam granites from the CSZ show moderate $\mathrm{SiO}_{2}$ (70.1-74.0 wt.\%), $\mathrm{Al}_{2} \mathrm{O}_{3}$ (14.4-16.5 wt.\%), $\mathrm{Na}_{2} \mathrm{O}$ (2.0-5.2 wt.\%), $\mathrm{K}_{2} \mathrm{O}$ (3.36.2 wt.\%) contents and are similar to the Dharapuram granites. The granites near Aravakurchi area are slightly higher in $\mathrm{SiO}_{2}$ (72.4-76.1 wt.\%), $\mathrm{Na}_{2} \mathrm{O}$ (3.0-4.1 wt.\%) than the Dharapuram granite, which contain higher $\mathrm{Al}_{2} \mathrm{O}_{3}$ (av. 15.7 wt.\%) and lower amounts of $\mathrm{Na}_{2} \mathrm{O}$ (av. 4.27 wt.\%) and $\mathrm{K}_{2} \mathrm{O}$ (av. 4.1 wt.\%) compared to Kangayam, Palladam Tiruchengodu, Sankari and Udhiyur granites. Puliyampatti granites contain higher $\mathrm{SiO}_{2}$ (70.1-73.6 wt. $\%$, av. 72.79 wt. $\%$ ), $\mathrm{Al}_{2} \mathrm{O}_{3}$ (15.1-17.2 wt. $\%$, av. 15.85 wt. $\%), \mathrm{Na}_{2} \mathrm{O}(3.9-8.1$ wt $\%$, av. 6.5 wt. $\%$ ) and lower $\mathrm{MgO}$ (0.1 wt.\%), $\mathrm{CaO}$ (12.8 wt. $\%$, av. 2.2 wt. $\%), \mathrm{K}_{2} \mathrm{O}(0.5-5.2$ wt. $\%$, av. 1.9 wt.\%) than the Pollachi-Udumalpet granites (table 2). The Munnar, Madurai, Palani, Rayanpatti and Oddanchatram granites from the Madurai Block vary in $\mathrm{SiO}_{2}$ (68.0-73.2 wt.\%), $\mathrm{Al}_{2} \mathrm{O}_{3}$ (12.59-15.97 wt.\%) and $\mathrm{CaO}(1.3-3.32$ wt.\%) and they contain more alkalies.

Regarding trace elements concentrations, the Northern Block granites show more variation than the granites of CSZ (table 2) and these are poor in Rb (3-134 ppm), Ba (581-913 ppm), U (<1 ppm) and $\mathrm{Th}(<2 \mathrm{ppm})$, but $\mathrm{Sr}$ is moderate to high (303-872 ppm). In the CSZ, Sankari-Tiruchengodu granites contain moderate $\mathrm{Sr}$ (284-285 ppm), Rb (57-98 ppm) and Ba (578-746 ppm). The Kangayam and Palladam granites show higher $\mathrm{Sr}$ (392-440 ppm), Ba (1962-3298 ppm) and relatively moderate Rb (70-86 ppm) and Zr (127-136 ppm). The Aravakurchi granites also contain higher $\mathrm{Sr}$ (194-304 ppm), Zr (24-217 ppm), Ba (823-2971 ppm) and Th (up to $67 \mathrm{ppm}$ ). The PollachiUdumalpet granites contain higher in trace elements concentration Ga (12-50 ppm), Rb (16-151 ppm), Sr (66-1955 ppm), Y (4-19 ppm), Zr (11$256 \mathrm{ppm}), \mathrm{Nb}(1-26 \mathrm{ppm}), \mathrm{Ba}(290-5542 \mathrm{ppm})$, Th (0.4-124 ppm) and U (0.2-6 ppm) compared to the Puliyampatti granites (table 2). In the Madurai Block, the Palani, Oddanchatram granites show moderate Rb (47-87 ppm) Zr (46-793 ppm) and higher Sr (679-689 ppm) and Ba (1102-1822 ppm) concentrations. Madurai, Rayanpatti and Munnar granites also have higher concentrations of trace elements like Rb (90-241 ppm), Sr (29-366 ppm), Zr (106-1521 ppm), Ba (448-1191 ppm), Hf (3$16 \mathrm{ppm})$ and $\mathrm{Th}(7-263 \mathrm{ppm})$ compared to the granites of CSZ and Northern Block.

In the normative $\mathrm{An}-\mathrm{Ab}-\mathrm{Or}$ plot (figure 4a), these granites define a compositional trend from albite end to orthoclase end and are confined mostly of granite-granodiorite fields and rarely trondhjemite field (Veppanapalli and Kangayam). But, the Barugur granite plot in tonalite field and the granites of Jakkampatty and Aravakurchi plotted in granodiorite fields. The $\mathrm{R}_{1}$ and $\mathrm{R}_{2}$ relation (Roche et al. 1980), also suggests that (figure 4b) most of the granites from the CSZ are granodioritic to monzogranite in composition, whereas the Pollachi-Udumalpet granite ranges from syenite, syenodiorite and is syn- or late-orogenic signature (Mallikharjuna Rao et al. 2005). The Jakkmapatty granite plots in tonalite field. Many of the granites from the three blocks are having similar average $\mathrm{A} / \mathrm{CNK}$ ratios, but the individual plutons show considerable spread in their $\mathrm{A} / \mathrm{CNK}$ ratios. In binary $\mathrm{A} / \mathrm{NK}$ against $\mathrm{A} / \mathrm{CNK}$ plot (figure 5), the granite plots on either side of the demarcation line between metaluminous and slightly peraluminous suggesting that all the granites derived from a source of narrow chemical composition.

The REE patterns of the granitic plutons in Northern Block show wide range of rare earth elements (REE) and fractionation trends ( $\sum \mathrm{REE}$ 23-124 ppm, La $\left.{ }_{N} / \mathrm{Yb}_{\mathrm{N}}=10.2-67.5\right)$. These granites show the slight enrichment of LREE and exhibit positive $\mathrm{Eu}$ anomalies except Jakkampatty and Veppanapalli granites (figure 6a). The Veppanapalli granites show slightly higher concentration of HREE and a strong negative anomaly (av. $\mathrm{Eu}_{\mathrm{N}} / \mathrm{Eu}_{\mathrm{N}}^{*}=1.22$ ) (figure $4 \mathrm{a}$ ). The Jakkampatty granites also show enriched REE patterns (av. $\mathrm{La}_{N} / \mathrm{Yb}_{\mathrm{N}}=17.67$ ) and show minor negative Eu anomaly (av. $\mathrm{Eu}_{\mathrm{N}} / \mathrm{Eu}_{\mathrm{N}}^{*}=3.68$ ) (figure 6a). Both the LREE (av. $\mathrm{La}_{\mathrm{N}} / \mathrm{Sm}_{\mathrm{N}}=2.77$ ) and HREEs (avg. $\left.\mathrm{Gd}_{\mathrm{N}} / \mathrm{Yb}_{\mathrm{N}}=4.46\right)$ show almost flat trends of these two granitoids. The Vaniyambadi granites show strong fractionation trend and poorly concentrated $\operatorname{REE}\left(\sum \mathrm{REE}=\right.$ up to $\left.23 \mathrm{ppm}\right)$ with strong positive Eu anomaly (av. $\left.\mathrm{Eu}_{\mathrm{N}} / \mathrm{Eu}_{\mathrm{N}}^{*}=4.96\right)$ compared to the other granites like Veppanapalli $\left(\sum \mathrm{REE}=\right.$ 107-142 ppm) and Jakkampatty ( $\sum$ REE $=128$ $343 \mathrm{ppm})$. The Krishnagiri granites also show variations in total $\mathrm{REE}$ content $\left(\sum \mathrm{REE}=6.4\right.$ $214 \mathrm{ppm}$ ) and they also exhibit clear fractionation trend (figure 6a; av. $\mathrm{La}_{\mathrm{N}} / \mathrm{Yb}_{\mathrm{N}}=14.13$ ) and (av. $\left.\mathrm{Gd}_{\mathrm{N}} / \mathrm{Yb}_{\mathrm{N}}=1.45\right)$ with Eu positive anomaly. 
a

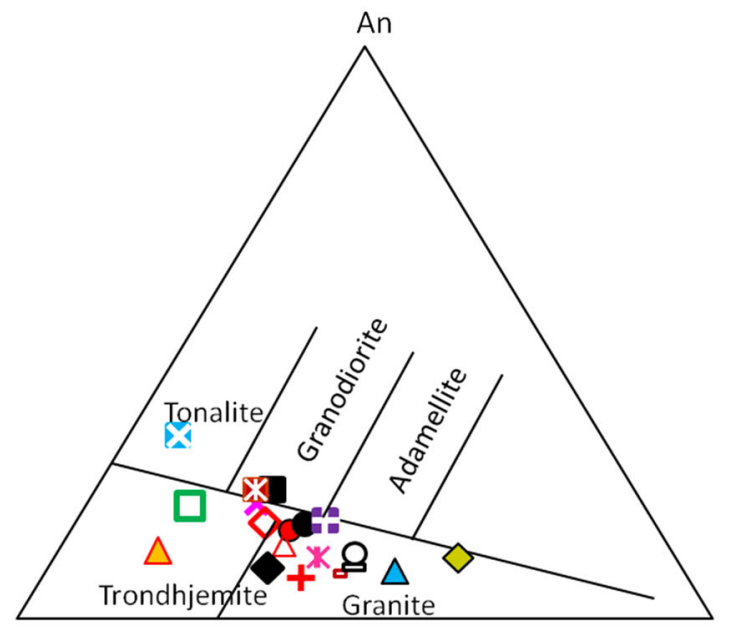

$\mathrm{Ab}$

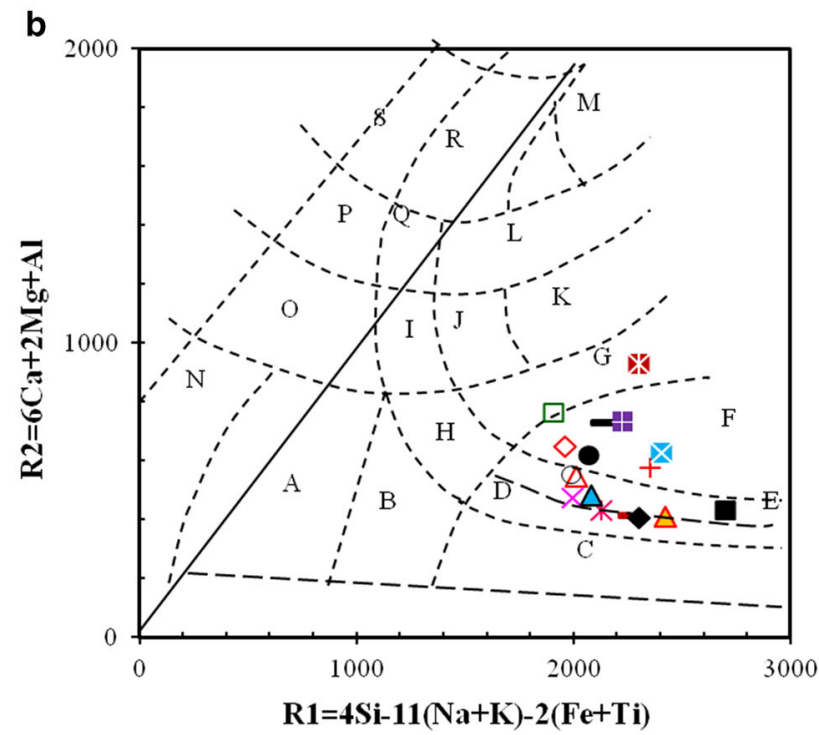

Figure 4. (a) Normative An-Ab-Or plot for SGT granitoid (after O'Connor 1965) and (b) The $\mathrm{R}_{1}-\mathrm{R}_{2}$ classification diagram (after De la Roche et al. 1980), the fields are defined as follows. A: Syenite, B: Quartz syenite, C: Alkali granite, D: Monzonite, E: Monzogranite, F: Granodiorite, G: Tonalite, H: Quartz monzonite, K: Diorite, L: Gabbro, M: Granodiorite, N: Nepheline Syenite, O: Syenodiorite, P: Syenogabbro, Q: Monzogabbro, R: Olivine Gabbro, S: Alkaline Gabbro, symbols as in figure 3 .

The Burgur granites are poorly enriched in REE $\left(\sum \mathrm{REE}=23-51 \mathrm{ppm}\right)$ and show strong positive Eu anomaly (av. Eu/Eu* $=3.58$ ) comparatively. The strong positive Eu anomaly of these granites may be due to plagioclase rich source (figure 6a). However, the slight increase in HREE of Vaniyambadi, Veppanapalli and Krishnagiri granites may be due to accessory minerals like zircon and monazites (figure 6a).

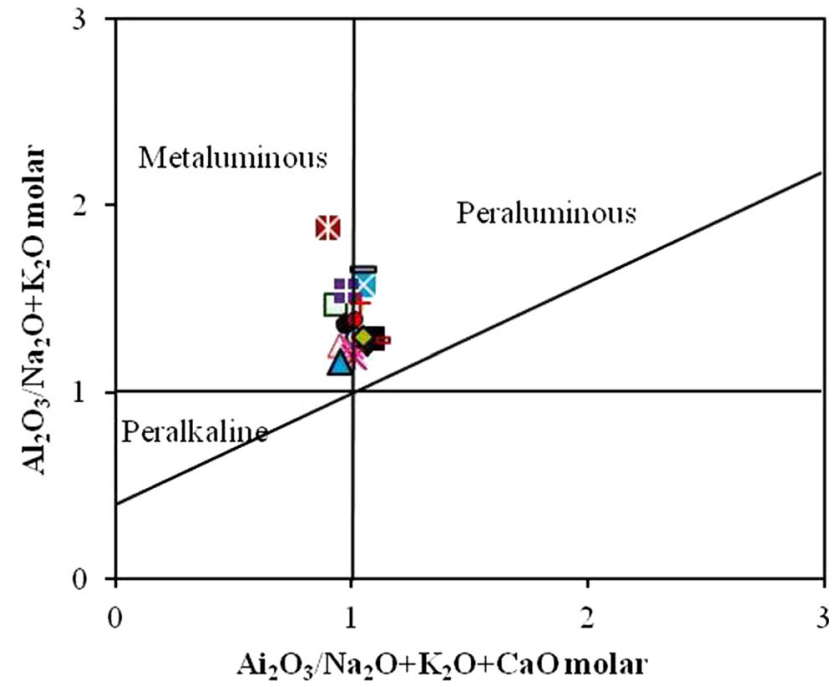

Figure 5. Binary A/NK vs. A/CNK diagram showing metaluminous to slightly peraluminous character of granites (after Maniar and Piccoli 1989), symbols as in figure 3.

The CSZ granites show REE considerable variation. The europium anomaly $\mathrm{Eu}_{\mathrm{N}} / \mathrm{Eu}_{\mathrm{N}}^{*}$ varies from 2.2.8 to 4.22 and show slight enriched patterns of LREE (av. $\mathrm{LaN}_{\mathrm{N}} / \mathrm{Sm}_{\mathrm{N}}=2.77-9.95$ ) and flat HREE (av. $\mathrm{Gd}_{\mathrm{N}} / \mathrm{Yb}_{\mathrm{N}}=1.12-14.35$ ) (figure $6 \mathrm{~b}$ ). The Kangayam granites show moderate concentration of REE ( $\sum \mathrm{REE}=15-107 \mathrm{ppm}$, av. LaN/ $\left.\mathrm{Yb}_{\mathrm{N}}=7.16\right)$ and exhibit slight Eu anomaly $\left(\right.$ av. $\mathrm{Eu}_{\mathrm{N}} / \mathrm{Eu}_{\mathrm{N}}^{*}=3.66$ ) (figure $6 \mathrm{~b}$ ). Palladam granites show similar to Kangayam granites with positive Eu anomaly (av. $\mathrm{Eu}_{\mathrm{N}} / \mathrm{Eu}_{\mathrm{N}}^{*}=4.19$ ) and HREE is moderately enriched (av. $\mathrm{Gd}_{\mathrm{N}} / \mathrm{Yb}_{\mathrm{N}}=1.69$ ). The Puliyampatti granites show eniriched REE $\left(\sum \mathrm{REE}=2.6-30.8\right.$, av. 18.3 ppm, av. $\mathrm{La}_{\mathrm{N}} / \mathrm{Yb}_{\mathrm{N}}$ $=15.11$ ), enriched LREE (av. $\mathrm{La}_{\mathrm{N}} / \mathrm{Sm}_{\mathrm{N}}=2.46$ ) and depleted HREE (av. $\mathrm{Gd}_{\mathrm{N}} / \mathrm{Yb}_{\mathrm{N}}=3.29$ ) and a strong positive $\mathrm{Eu}$ anomaly $\left(\mathrm{av} . \mathrm{Eu}_{\mathrm{N}} / \mathrm{Eu}_{\mathrm{N}}^{*}=2.84\right.$; figure 6b). Udhiyur granites have varied similar REE patterns ( $\sum$ REE 2-78 ppm) and exhibit possitve Eu anomaly (av. Eu $\left./ \mathrm{Eu}_{\mathrm{N}}^{*}=2.99\right)$ similar to Puliyampatti granites. The Europium anomalies from these plutons may be due to non-removal of plagioclases from the melt compositions. In Pollachi-Udumalpet granites, majority of samples show enriched REE (around $600 \mathrm{ppm}$ ) with some abnormalities like very low or high concentration of $\operatorname{REE}\left(\sum \mathrm{REE}=24-3432 \mathrm{ppm}\right.$, av. 582 $\mathrm{ppm}$ ) and fractionated trends (av. $\mathrm{La}_{\mathrm{N}} / \mathrm{Sm}_{\mathrm{N}}=$ 9.95 and av. $\mathrm{Gd}_{\mathrm{N}} / \mathrm{Yb}_{\mathrm{N}}=3.83$ ) with slight positive $\mathrm{Eu}$ anomalies (av. $\mathrm{Eu}_{\mathrm{N}} / \mathrm{Eu}_{\mathrm{N}}^{*}=4.22$; figure $6 \mathrm{~b})$. The Tiruchengodu granites are moderately enriched in $\operatorname{REE}\left(\sum \mathrm{REE}=48-727 \mathrm{ppm}\right)$ 


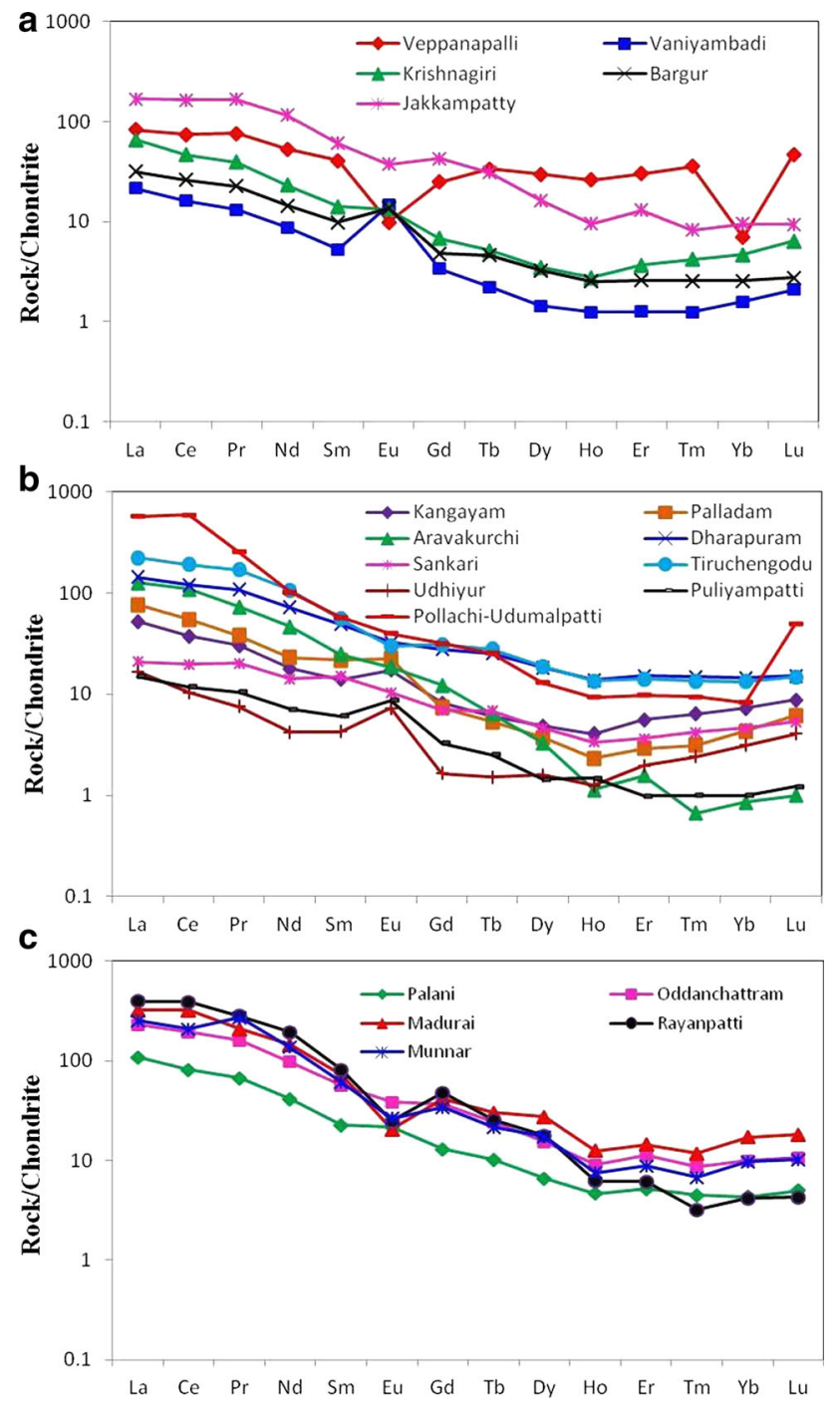

Figure 6. Rare earth element pattern of different granite plutons (averages): (a) Northern Block, (b) CSZ and (c) Madurai Block; normalized values are after Sun and Mc donough (1989).

and show fractionation trends (av. $\mathrm{La}_{\mathrm{N}} / \mathrm{Yb}_{\mathrm{N}}=$ 16.93) and exhibit negative Eu anomaly on average (av. $\mathrm{Eu}_{\mathrm{N}} / \mathrm{Eu}_{\mathrm{N}}^{*}=3.21$; figure $\left.6 \mathrm{~b}\right)$. The Dharapuram granites are also similar to Tiruchengodu granites with enriched REE ( $\sum \mathrm{REE}=35.7-$ $558.2 \mathrm{ppm}$ ) and show negative Eu anomaly on average $\left(\mathrm{av} . \mathrm{Eu}_{\mathrm{N}} / \mathrm{Eu}_{\mathrm{N}}^{*}=3.69\right)$. The Sankari granites are poorly enriched in REE ( $\sum \mathrm{REE}=$ 18.41-52.62 ppm) and show slight flat pattern with negative europium anomalies $\left(\mathrm{Eu}_{\mathrm{N}} / \mathrm{Eu}_{\mathrm{N}}^{*}=0.75-2\right.$, av. 2.21) on average. The Aravakurchi granites show fractionation trend and enrichment of REE $\left(\sum \mathrm{REE}=39-208, \mathrm{Eu}_{\mathrm{N}} / \mathrm{Eu}_{\mathrm{N}}^{*}=132 \mathrm{ppm}\right)$ without much significance of negative $\mathrm{Eu}$ anomaly $\left(\right.$ av. $\mathrm{Eu}_{\mathrm{N}} / \mathrm{Eu}_{\mathrm{N}}^{*}=3.04$; figure $\left.6 \mathrm{~b}\right)$. In general, the granites of Sankari, Udhiyur, Kangayam and Puliayampatti are very poor in REE contents compared to other granites of CSZ.

From the Madurai Block, in the Palani and Oddanchatram granites, the av. REE concentration varies between 111 and $258 \mathrm{ppm}\left(\mathrm{av} . \mathrm{La}_{\mathrm{N}} / \mathrm{Yb}_{\mathrm{N}}\right.$ =18.6-25.36) with enrichment of LREE (av. LaN/ $\mathrm{Sm}_{\mathrm{N}}=4-4.8$, av. $\left.\mathrm{Gd}_{\mathrm{N}} / \mathrm{Yb}_{\mathrm{N}}=2.3-3.7\right)$. The Palani granites are moderately enriched in REE ( $\sum$ REE $=111 \mathrm{ppm}$ ) and shows positive Eu anomaly (av. $\mathrm{Eu}_{\mathrm{N}} / \mathrm{Eu}_{\mathrm{N}}^{*}=3.6$; figure $6 \mathrm{c}$ ). The Oddanchatram granites are strongly enriched in REE ( $\sum \mathrm{REE}=$ 127.7-606.8 ppm) and show strong fractionation trends $\left(\right.$ av. $\mathrm{La}_{\mathrm{N}} / \mathrm{Yb}_{\mathrm{N}}=23.11$ ) with minor $\mathrm{Eu}$ anomaly (av. $\mathrm{Eu}_{\mathrm{N}} / \mathrm{Eu}_{\mathrm{N}}^{*}=3.95$; figure $6 \mathrm{c}$ ). The Madurai granites show (figure 6c) similar REE concentration and strongly enriched REE contents $\left(\sum \mathrm{REE}=48.2-687 \mathrm{ppm}\right)$ respectively and show strong negative Eu anomaly (av. $\mathrm{Eu}_{\mathrm{N}} / \mathrm{Eu}_{\mathrm{N}}^{*}=$ 1.95) compared to Palani and Oddanchatram granites. The Munnar and Rayanpatti granites are also enriched in REE contents $\left(\sum \mathrm{REE}=135.6^{-}\right.$ $\left.917.2 \mathrm{ppm}, \sum \mathrm{REE}=37.6-884.5 \mathrm{ppm}\right)$ with depleted HREE (av. $\mathrm{Gd}_{\mathrm{N}} / \mathrm{Yb}_{\mathrm{N}}=3.5-11.4$ ) and show strong negative $\mathrm{Eu}$ anomaly (av. $\mathrm{Eu}_{\mathrm{N}} / \mathrm{Eu}_{\mathrm{N}}^{*}=$ 2.71, av. $\mathrm{Eu}_{\mathrm{N}} / \mathrm{Eu}_{\mathrm{N}}^{*}=2.91$; figure $6 \mathrm{c}$ ). The early formed plagioclase removal from the early melts may cause for the observed strong negative Eu anomalies and showing strong alkaline character of the source of these granites.

\section{Discussion}

Generation of granitic magma is associated in space and time with the growth of the continental crust, rather than just recycling (Patino Douce 1999). Granites can result from partial fusion of continental crustal rocks at low pressure, without visible contribution of mafic magma. Partial melting of mantle peridotite produces basaltic magma that can undergo extensive fractional crystallization to produce granitic (non-orogenic) peralkaline granitoids and most Proterozoic TTG suites. Petrology and geochemical characteristics of granitic rocks from the study area are syenogranite to monozogranite in composition and few of them show alkali feldspathic syenite to synetic composition. Quartz, K-feldspar and plagioclase are the major phases with ferromagnesian minerals like hornblende and biotite as accessories in these granites. The A/CNK molecular ratios of these granites range between 0.91 and 1.2 with predominantly 
metaluminous character and I-type to S-type signatures (figure 5; Chappell and White 1974, 1992). The source for the I-type metaluminous granites are possibly mafic and metaigneous rocks (Chappell and White 1974; Frost et al. 2001), and for granites of slightly peraluminous nature (S-type), probably pure crustal melts that are uncontaminated by mantle material (Castro et al. 1999b; Ghani et al. 2013; El-bialy and Omar 2015). It is well described that the radiogenic and stable isotopic compositions of peraluminous leucogranites, their common field settings within mediumto high-grade regional metamorphic terrains show lack of spatial and temporal association between them, and the experimental studies have shown that melting of metasedimentary rocks generates peraluminous silica-rich melts (Patino Douce and Harris 1998). The slightly peraluminous and the metaluminous nature of these granites suggests their derivation from either melting of mafic rocks or amphibolites (Ellis and Thompson 1986; Patino Douce 1999). The REE patterns of these granites from all the three blocks show broad variations (figure $6 \mathrm{a}-\mathrm{c}$ ). The granites of Northern Block show both positive and negative Eu anomalies with moderately enriched REE contents and their sources may relate to melting of undifferentiated mantlederived rocks. The Veppanapalli and Jakkampatty granites from this block show strong negative $\mathrm{Eu}$ anomalies that resemble the melts of upper continental crust. Vaniyambadi, Krishnagiri and Barugur granites show positive Eu anomalies that represent restites after partial melting (Rundick 1991). The REE patterns of the granites from CSZ suggest different modes of origin and evolutionary history. They show both positive and negative $\mathrm{Eu}$ anomalies of mixed characteristics. There is depletion of REE in the granites of Kangayam $\left(\sum \mathrm{REE}=14-106\right.$, av. $\left.=54 \mathrm{ppm}\right)$ Palladam $\left(\sum \mathrm{REE}=10-184\right.$, av.$\left.=73 \mathrm{ppm}\right)$, Puliyampatti $\left(\sum \mathrm{REE}=2.6-31\right.$, av. $\left.=18.3 \mathrm{ppm}\right)$, Udhiyur $\left(\sum \mathrm{REE}=1.87-78.14\right.$, av. $\left.=15 \mathrm{ppm}\right)$ and show slight positive Eu anomalies that resemble possibly early intruded plutons of I-type. This positive Eu anomaly in these granites is due to feldspar accumulation or assimilation of feldspar-rich material (Ragland 1989). However, the granites of Madurai Block are highly enriched in REE ( $\sum \mathrm{REE}=111-$ $3853 \mathrm{ppm}$ ) and exhibit strong fractionation trends with significant negative $\mathrm{Eu}$ anomalies (except for Palani) and show fractionated LREE with moderate enrichment of HREE that suggests they might have been generated from deeper source or due

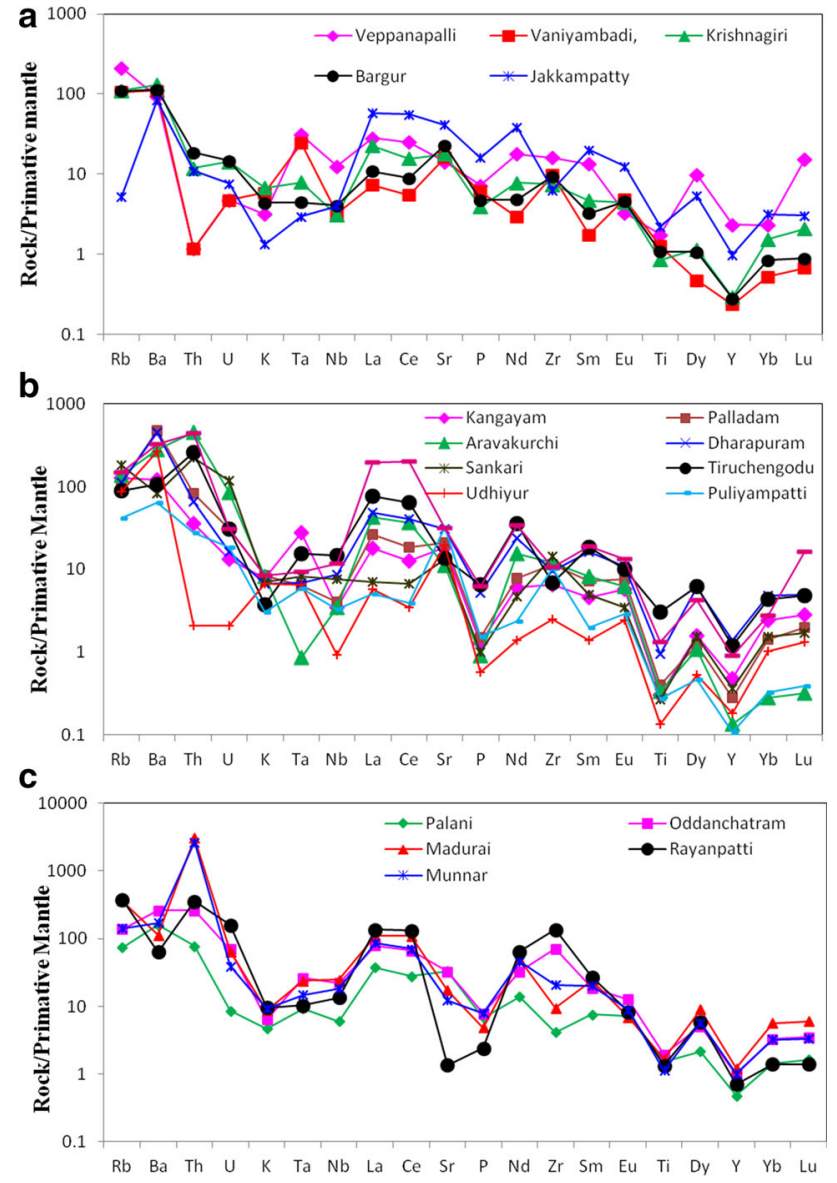

Figure 7. Rock/primative mantle spider plot of different granite plutons (average): (a) Northern Block, (b) CSZ and (c) Madurai Block; normalized values (after, Sun and Mc donough 1989).

to addition of juvenile magmas, and data also reflect crustal contamination of source magma. The variations in REE concentrations and enrichment may be due to accessory minerals like allanite, monazite, sphene and zircon contents in the rock. In the primitive mantle-normalized spider plot (figure $7 \mathrm{a}-\mathrm{b})$, many of the granites from the Northern block and CSZ (Kangayam, Palladam, Puliyampatti, Udhiyur) show positive $\mathrm{Rb}, \mathrm{Ba}, \mathrm{Sr} \mathrm{Th}$, and light REEs anomalies and negative $\mathrm{Nb}, \mathrm{P}$ and $\mathrm{Ti}$ anomalies resembling the characteristics of the average continental arcs (Condie and Kroner 2013). These high $\mathrm{Ba}, \mathrm{Sr}$ granitoids are interpreted as tectonic regimes of elevated geotherms, including ridge subduction, rifting or subduction of young hot oceanic crust (Whalen et al. 1997). However, Madurai block granites and some of the granites of CSZ (Sankari, Tiruchengodu, Dharapuram, Aravakurchi and Pollachi-Udumalpet) show enrichment of $\mathrm{Rb}, \mathrm{Th}, \mathrm{U}$ and depleted $\mathrm{Ti}, \mathrm{Sr}$, 
$\mathrm{P}$, Eu anomalies (figure 7c) that resemble A-type characteristics (David and Chappell 1992).

Many distinguishing features have been outlined by Chappell and White (1974) for classifying granitoids into I- and S-types and for deciphering the tectonic environment. Several schemes and methods have been proposed by Pearce et al. (1984) and Maniar and Piccoli (1989). The scheme of Maniar and Piccoli (1989) indicates that the granitoid compositions are consistent with island arc, continental arc or continental collision. Tectonic discrimination diagrams of these granites show some of these are confined to volcanic arc granite types and show syn-collisional characteristics and some show postcollisional characterstics (Peace 1996). In spite of their chemical coherence on some variation diagrams (figure 8a-d), the plutons collectively exhibit incoherent trends for some major and trace elements. In the $\mathrm{Rb}$ vs. $(\mathrm{Y}+\mathrm{Nb}), \mathrm{Nb}$ vs. $\mathrm{Y}, \mathrm{Rb}$ vs. $(\mathrm{Yb}+\mathrm{Ta})$ binary variation diagrams (figure $8 \mathrm{a}-$ c), some of the granites (Vaniyambadi, Bargur, Krishnagiri, Kangayam, Palladam, Udhayur, Pulliyampatti and Palani are confined to the volcanic arc to syncollisional granite fields, and their genesis might have been associated with the Neoarchean and Neoproterozoic subduction-accretion tectonics described in northern margin of terrain and that of CSZ and south of Madurai block (Yellappa et al. 2012; Santosh et al. 2009, 2012, 2013, 2015, 2017; Ratheesh Kumar et al. 2016; Bhadra and Nasipuri 2017). It is also described that the basement gneisses and intrusive granitic rocks near the transition zone around Krishnagiri as Itype and S-type (Chandrasekaran and Subrmanian 1996). The geochemical signatures of Veppanapalli, Vaniyambadi, Bargur and Jakkampatty granites from the present study are similar to Krishnagiri granites. The granites from the CSZ (Sankari, Tiruchengodu, Pollachi-Udumalpet, Dharapuram and Aravakurchi and Madurai Block (Munnar, Madurai, Oddenchetram and Rayanpatti) granites show syn-collisional to post-tectonic settings (figure 8a, d) and their evolution might be associated with extensional tectonics after post-collision. In $\mathrm{Zr}$ vs. Ga/Al diagram (figure 9a), majority of the granites from the present study show fractionated I-type, S-type and some are confined to A-type fields (Madurai, Rayanpatti, Munnar and Oddanchatram). Similarly in $\mathrm{FeO}^{*} / \mathrm{MgO}$ vs. $\mathrm{Zr}+\mathrm{Nb}+\mathrm{Ce}+\mathrm{Y}$ diagram (figure $9 \mathrm{~b}$ ), most of the granites show fractionated I-type, while some like Sankari, Pollachi-Udumalpet, Oddanchatram, Madurai, Rayanpatti, and Munnar show A-type nature. Earlier studies have established (Condie et al. 1982, 1986; Dhanaraju et al. 1983; Chandrasekharan 2002) that the Sankari-Tiruchengodu granites from the CSZ are post-tectonic, calcalkaline and slightly peraluminous in nature (Nathan et al. 2001). It is also described based on geochemical and mineralogical characterstics that the granites of Sankari-Tiruchengode are S-type and formed in continental collision tectonic setting showing later aborted rifting (Nathan et al. 1994). Recently, the granites of Sankari, Tiruchengodu, Puliyampatti, Karamadai and Maruda Malai from the CSZ are described as A-type granites (GSI, 2009). A-type granitic melts occur in many continents and are of different ages and occur in a variety of tectonic settings (Whalen et al. 1987). These rocks result mainly by partial melting of $\mathrm{F}$ and/or Cl-enriched, dry granulitic residue remaining in the lower crust after extraction of anorogenic granites (Whalen et al. 1987). The origins of A-type granites were originally defined as anorogenic nature (Loiselle and Wones (1979). Later, it was also described that they can be formed in both anorogenic and post-orogenic settings (Whalen et al. 1987; Eby 1992). Geochemical characters of the granites from the Madurai block and some of granite of CSZ (described above) are broadly comparable with the A-type granites which indicated their possible post-orogenic nature. It is described that the granites south of Madurai at Ambasamudram and Rajapalayam intrude into the high-grade rocks showing the characteristics of Atype granites (Pandey et al. 1994). The granitoids at Kullampatti area, Salem district near Sankari and leucogranites of pegmatoidal granites within the CSZ were also described as A-type origin (Roy and Dhanaraju 1999; Nathan et al. 2001). From the present study the geochemical characteristics of Munnar, Rayanpatti and Oddanchatram of Madurai clearly show their A-type origin (figure 9a-b) in confirmation with the results of several other workers (Santosh and Nair 1983; Santosh et al. 1993; Thampi et al. 1993; Rajesh and Santosh 1996; Rajesh 2008).

It is well established that, the basement gneisses and intrusive granitic rocks occurring near the transition zone around Krishnagiri in the northern part of Tamil Nadu are predominantly of tonalitic-granodioritic in composition, Archean in age and are believed to have formed by partial melting of garnet-bearing amphibolites (Condie et al. 1982, 1986; Dhanaraju et al. 1983; Chandrasekharan 2002). It is also described that the 

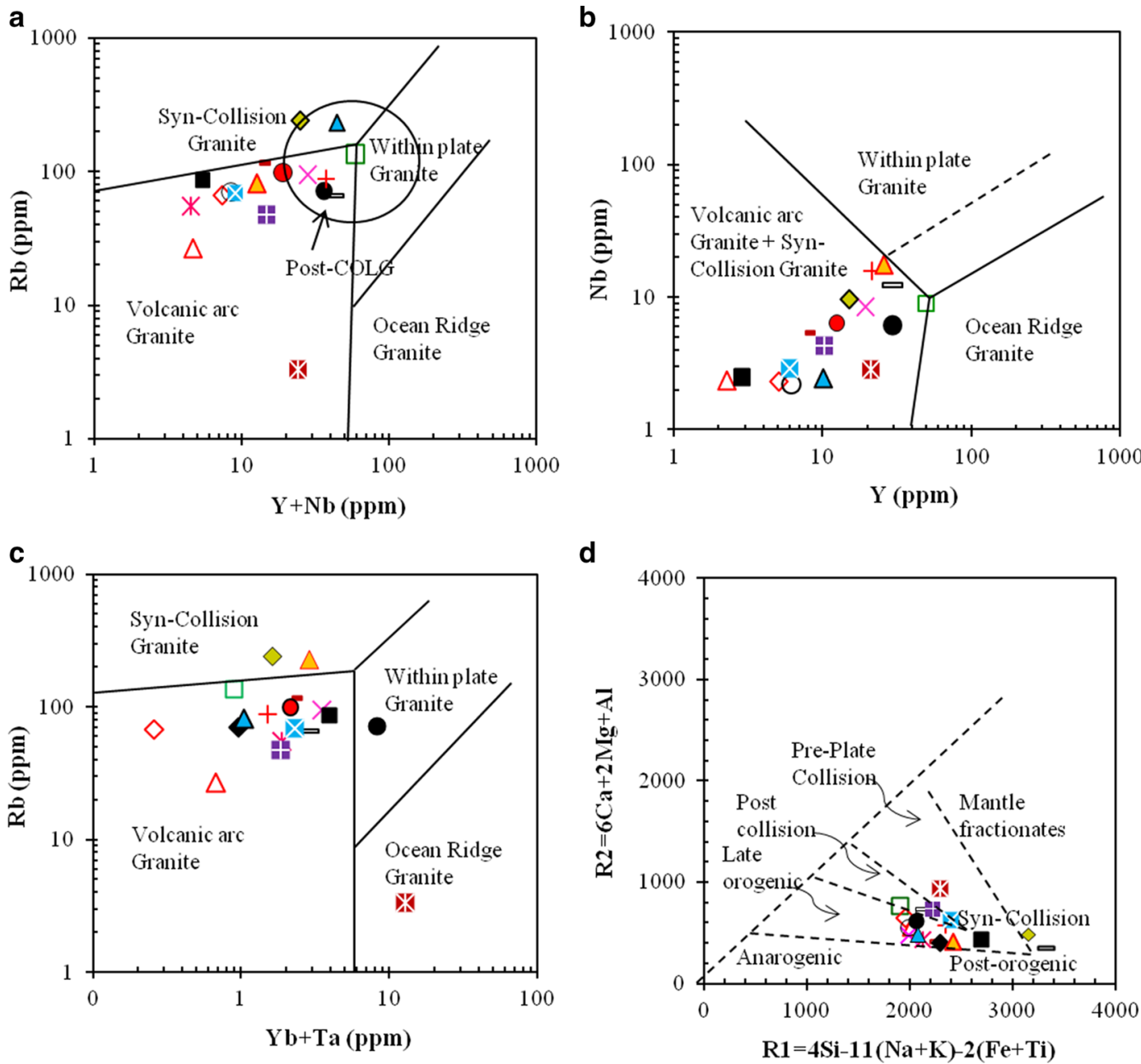

Figure 8. The tectonic discrimination diagrams: (a) Rb vs. ( $\mathrm{Y}+\mathrm{Nb})$, (b) Nb vs. $\mathrm{Y}$, (c) Rb vs. (Yb+Ta) for different granite plutons (after Pearce et al. 1984; Pearce 1996), and (d) $\mathrm{R}_{1}-\mathrm{R}_{2}$ tectonic classification diagram of plutonic rocks (after Batchelor and Bowden 1985) $\mathrm{R}_{1}=4 \mathrm{Si}-11(\mathrm{Na}+\mathrm{K})-2(\mathrm{Fe}+\mathrm{Ti}) ; \mathrm{R}_{2}=6 \mathrm{Ca}+2 \mathrm{Mg}+\mathrm{Al}$; symbols as in figure 3 .

pink granitic suite which is well-differentiated, calc-alkaline, metaluminous to mild peraluminous, late-kinematic granitoids is evolved by anatectic remelting of grey granitoids (Chandrasekharan 1996). The origin also implies for the evolution of several potash-rich feldspar granites during Archean-Proterozoic transition (Chandrasekharan 1996). The different granites in the SGT like Vaniyambadi, Bargur, Krishnagiri, Palladam, Kangayam and Puliyampatti granites show +ve Eu anomalies suggesting that they may have affinity towards crustal component of TTG assemblages with subduction-related process, while the Veppanapalli, Jakkampatty, Aravakurchi, PollachiUdumalpet, Dharapuram, Rayanpatti, Munnar, and Madurai granites show nagative Eu anomaly without much involvement of earlier TTG component and seem to be products of crustal anetectic melting after the Neoproterozoic post-collision. The generation of granite melts is at relatively shallow depths and temperatures in the order of $600-900^{\circ} \mathrm{C}$ is recorded in experimental studies on silicate systems (Bonin et al. 2002). The Rb/Sr ratio is one of the indicators for magmatic differentiation, where it increases with higher degree of differentiation. The $\mathrm{Rb}$ vs. Sr plot (figure 9c) shows these granites have been derived from highly differentiated and more evolved granitic liquids of deeper origin (Condie 1973). Similarly, the curved trend of these granites on $\mathrm{Al}_{2} \mathrm{O}_{3} / \mathrm{TiO}_{2}$ vs. $\mathrm{TiO}_{2}$ (figure 9d) diagram represents well defined magmatic differentiation (Sun and Nesbitt 1978). However, in the normative $\mathrm{Q}-\mathrm{Ab}-\mathrm{Or}$ plot (figure 10), Puliyampatti granite indicates a calc-alkaline trend of crystallization temperatures between 750 and $800^{\circ} \mathrm{C}$, whereas the Pollachi-Udumalpet granitoids show wider scatter and range of crystallization temperatures between 700 and $800^{\circ} \mathrm{C}$ (Mallikharjuna Rao et al. 2005). The rest of the granite plutons in all the three tectonic blocks namely 
a

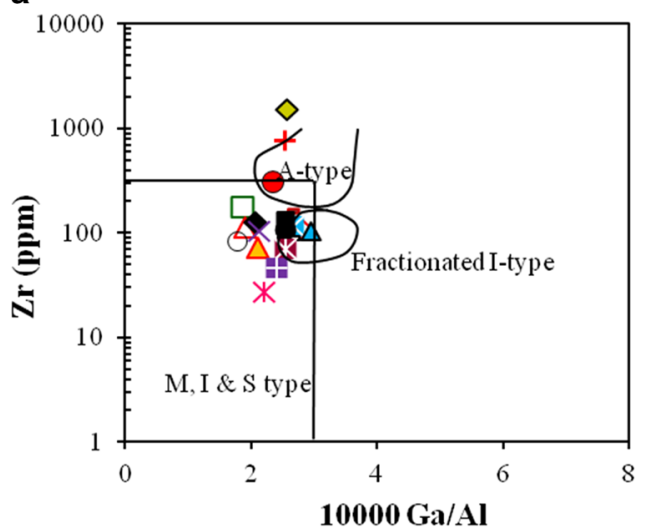

C

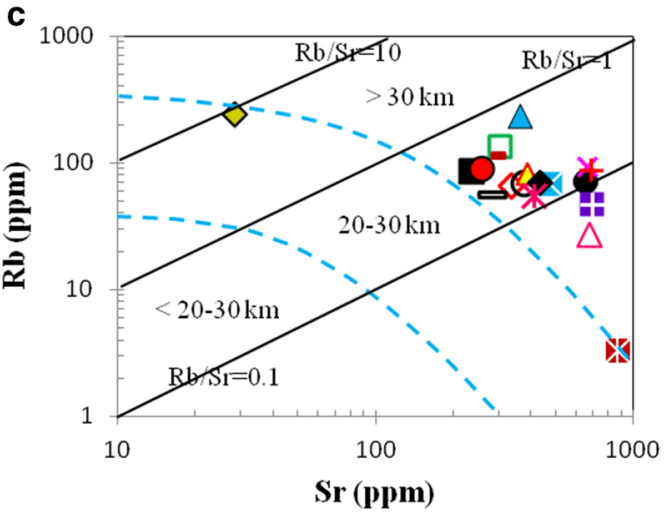

b

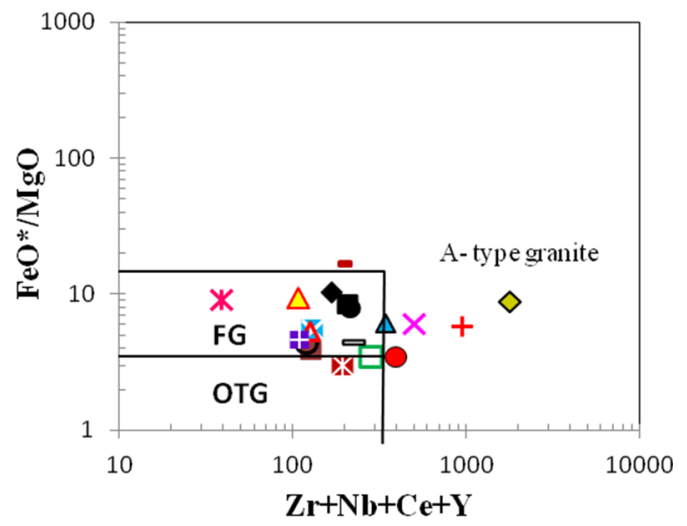

d

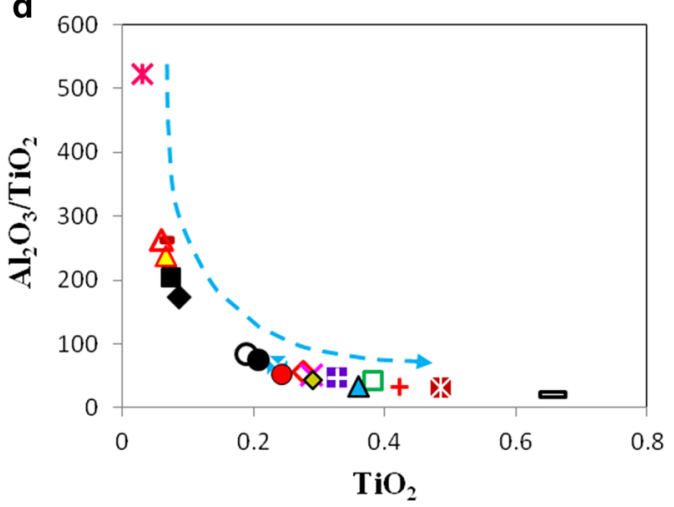

Figure 9. The tectonic discrimination diagrams: (a) Binary $\mathrm{Zr}$ vs. Ga/Al diagram showing I- to A-type nature of granitic rocks (after Whalen et al. 1987); (b) $\mathrm{FeO}^{*} / \mathrm{MgO}$ vs. $\mathrm{Zr}+\mathrm{Nb}+\mathrm{Ce}+\mathrm{Y}$ diagram (after Whalen et al. 1987); (c) Rb-Sr binary diagram, the dashed lines refer to the crustal thickness (after Condie 1973); (d) $\mathrm{Al}_{2} \mathrm{O}_{3} / \mathrm{TiO}_{2}$ vs. TiO 2 magma differentiation diagram (after Sun and Nesbitt 1978); symbols as in figure 3.

Veppanapalli, Vaniyambadi, Krishnagiri, Sankari, Udhiyur, Aravakurchi, Palani, Oddanchatram and Madurai show between 700 and $760^{\circ} \mathrm{C}$ temperature for their crystallization of melts (figure 10). These lower temperatures might be the addition of more water or carbon dioxide during melt generation. Similar temperature range $\left(650-700^{\circ} \mathrm{C}\right.$ under $5 \mathrm{~kb} \mathrm{P}_{\mathrm{H} 2 \mathrm{O}}$ ) of origin for potash feldspar granites in Northern Block is discussed by earlier workers (Chandrasekharan 1996).

\subsection{Age constraints}

The SGT has a complex evolutionary history from the early Archean to late Neoproterozoic (3500$550 \mathrm{Ma})$ with repeated multiple deformations, anatexis, intrusions and polyphase metamorphism. The summary of the available ages of granitoids from different tectonic blocks is given in table 3 . The granites and tonalites of $2.5 \mathrm{Ga}$ are common in the area of transition from amphibolite facies rocks of the Dharwar craton to the granulite facies rocks of the SGT as well as in the eastern

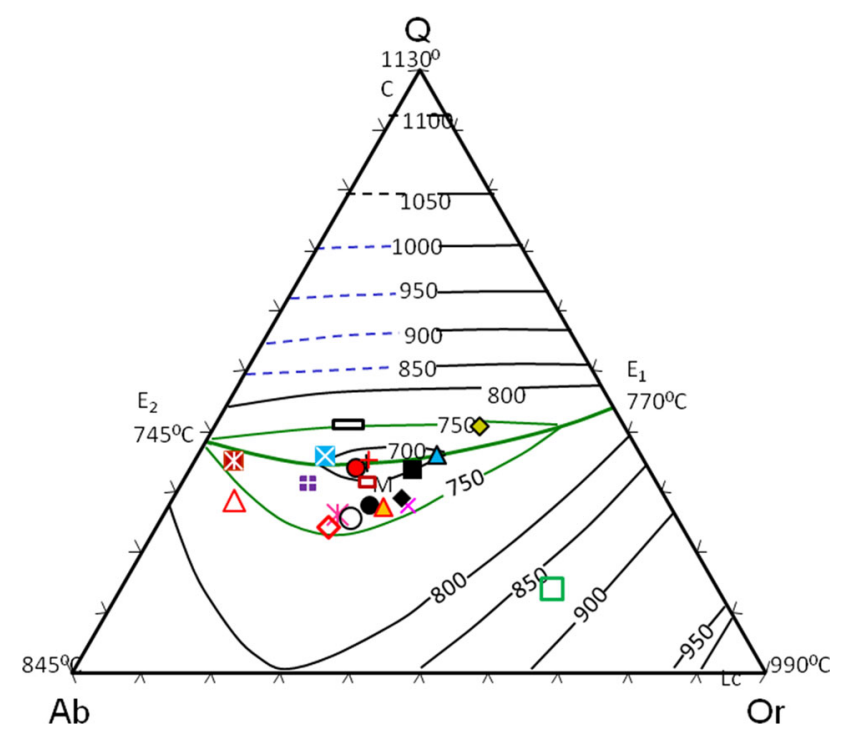

Figure 10. The normative $\mathrm{Q}-\mathrm{Ab}-\mathrm{Or}$ ternary diagram (after Tuttle and Bowen 1958) showing crystallization temperature of different granitoid melts; symbols as in figure 3 .

Dharwar Block (Peucat et al. 1993). These granites extend across the major tectonic shear zones and occur at Salem, Namakkal, Karur, Mettupalliyam 
Table 3. Brief summary of available ages of different granitoids in major tectonic blocks of the SGT.

\begin{tabular}{|c|c|c|c|c|}
\hline Rock type & Method/technique & Age (Ma) & Interpretation & Reference \\
\hline \multicolumn{5}{|l|}{ Northern Block } \\
\hline Gneisses & $\mathrm{U}-\mathrm{Pb}$ zircon & $2963 \pm 4$ & Protolith age & Friend and Nutman (1991) \\
\hline Granite & $\mathrm{U}-\mathrm{Pb}$ Zircon & 2513 & $\begin{array}{l}\text { Crystallization } \\
\text { age }\end{array}$ & Friend and Nutman (1991) \\
\hline Granite-gneiss & $\mathrm{Sm}-\mathrm{Nd}$ & 3130 & $\begin{array}{l}\text { Crystallization } \\
\text { age }\end{array}$ & Meissner et al. (2002) \\
\hline Migmatitic gneiss & Nd-TDM model age & 2780 & $\begin{array}{l}\text { Crystallization } \\
\text { age }\end{array}$ & Peucat et al. (2013) \\
\hline Krishnagri granite & $\mathrm{U}-\mathrm{Pb}$ zircon & $2557 \pm 16$ & Prootlith age & Peucat et al. (1993) \\
\hline Gingee granite & $\mathrm{K}$-Ar dating & $2250 \pm 76$ & $\begin{array}{l}\text { Crystallization } \\
\text { age }\end{array}$ & $\begin{array}{l}\text { GSI }(1978) \text {, } \\
\text { Balasubrahmanyan et al. } \\
(1979)\end{array}$ \\
\hline Sholingar & $\mathrm{K}$-Ar dating & $\sim 2302$ & Protolith age & $\begin{array}{l}\text { Krogstad and Hanson (1988), } \\
\text { Balasubrahmanyan and } \\
\text { Sarkar (1981) }\end{array}$ \\
\hline Gingee pluton & $\mathrm{K}$-Ar dating & $\sim 2254$ & Protolith age & - do- \\
\hline $\begin{array}{l}\text { Pink Granite } \\
\text { (Biligiri Rangan } \\
\text { Hills) }\end{array}$ & $\mathrm{U}-\mathrm{Pb}$ Zircon & $2490 \pm 21$ & $\begin{array}{l}\text { Crystallization } \\
\text { age }\end{array}$ & Ratheesh Kumar et al. (2016) \\
\hline \multicolumn{5}{|l|}{ Nilgiri Block } \\
\hline Enderbite gneiss & Sm-Nd model ages & $\sim 2620$ & Protolith age & $\begin{array}{l}\text { Kohler and Srikantappa } \\
\text { (1996) }\end{array}$ \\
\hline Ambalavayal granite & $\mathrm{Rb}-\mathrm{Sr}$ isochron & $595 \pm 20$ & Emplacement age & Santosh et al. (1986) \\
\hline Kalpatta granite & $\mathrm{U}-\mathrm{Pb}$ zircon & $553 \pm 5$ & Emplacement age & Miller et al. (1996) \\
\hline \multicolumn{5}{|l|}{ Salem Block } \\
\hline $\begin{array}{l}\text { Granite gneiss } \\
\text { (Salem) }\end{array}$ & TIMS U-Pb zircon & $2528 \pm 2$ & Emplacement age & Ghosh et al. (2004) \\
\hline \multirow[t]{2}{*}{$\begin{array}{l}\text { Metamorphosed } \\
\text { granite (Salem) }\end{array}$} & $\mathrm{U}-\mathrm{Pb}$ zircon & $\sim 2650$ & $\begin{array}{l}\text { Protolith and } \\
\text { high-grade } \\
\text { metamorphism }\end{array}$ & Sato et al. (2011) \\
\hline & & $\sim 2440$ & & \\
\hline Tirukovilur & $\mathrm{K}$-Ar dating & $\sim 2254$ & $\begin{array}{l}\text { Crystallization } \\
\text { age }\end{array}$ & $\begin{array}{l}\text { Balasubrahmanyan et al. } \\
\quad(1979)\end{array}$ \\
\hline Kullamaptti granite & $\mathrm{Rb}-\mathrm{Sr}$ isochron & $534 \pm 15$ & Emplacement age & Pandey et al. (1993) \\
\hline Granite(Kanjimalai) & ${ }^{207} \mathrm{~Pb} /{ }^{204} \mathrm{~Pb}$ age & $\begin{array}{l}2647 \pm 11 \\
2442 \pm 20\end{array}$ & $\begin{array}{r}\text { Crystallization, } \\
\text { thermal event }\end{array}$ & Sato et al. (2011) \\
\hline $\begin{array}{l}\text { Quartzo-feldspathic } \\
\text { gneiss }\end{array}$ & U-Pb Zircon & $\begin{array}{l}2475 \pm 1.8 \\
2483 \pm 2.5\end{array}$ & Emplacement age & Saitoh et al. (2011) \\
\hline \multicolumn{5}{|c|}{ Cauvery Suture Zone (CSZ) } \\
\hline Granite (Namakkal) & TIMS U-Pb zircon & $2511 \pm 28$ & Emplacement age & Ghosh et al. (2004) \\
\hline $\begin{array}{r}\text { A-type granite } \\
\text { (Ayyermalai) }\end{array}$ & $\mathrm{U}-\mathrm{Pb}$ zircon & $\sim 590$ & $\begin{array}{l}\text { Magmatism } \\
\text { high-grade } \\
\text { metamorphism }\end{array}$ & Sato et al. (2012) \\
\hline $\begin{array}{l}\text { Rapakivi granite } \\
\text { (Tangalamvaripatti) }\end{array}$ & $\mathrm{U}-\mathrm{Pb}$ zircon & $819 \pm 26$ & Protolith ages & Sato et al. (2012) \\
\hline $\begin{array}{l}\text { Sankari, } \\
\text { Tiruchengodu } \\
\text { leucocratic } \\
\text { granites }\end{array}$ & $\mathrm{Rb}-\mathrm{Sr}$ isotope & $534 \pm 15$ & $\begin{array}{l}\text { Crystallization } \\
\text { age }\end{array}$ & Pandey et al. (1993) \\
\hline
\end{tabular}


Table 3. (Continued.)

\begin{tabular}{|c|c|c|c|c|}
\hline Rock type & Method/Technique & Age (Ma) & Interpretation & Reference \\
\hline $\begin{array}{l}\text { Idappadi } \\
\text { trondhjemite }\end{array}$ & $\mathrm{Rb}-\mathrm{Sr}$ isotope & $479 \pm 12$ & Protolith age & Ghosh et al. (1996) \\
\hline $\begin{array}{l}\text { Puliyampatti } \\
\text { (leucocratic } \\
\text { granite) }\end{array}$ & $\mathrm{K}$-Ar dating & $471 \pm 10$ & Protolith age & GSI (1978) \\
\hline $\begin{array}{l}\text { Marudamalai } \\
\text { granite }\end{array}$ & $\mathrm{Rb}-\mathrm{Sr}$ isotope & $619 \pm 35$ & Protolith age & Nathan et al. (2001) \\
\hline $\begin{array}{l}\text { Plagiogranite } \\
\text { (Manamedu) }\end{array}$ & $\mathrm{U}-\mathrm{Pb}$ Zircon & $\begin{array}{l}737 \pm 23 \\
513 \pm 4.6\end{array}$ & Crystallization age & Santosh et al. (2012) \\
\hline $\begin{array}{l}\text { Trondhjemite } \\
\text { (Devanur) }\end{array}$ & U-Pb Zircon & $2528 \pm 61$ & Crystallization age & Yellappa et al. (2012) \\
\hline Metagranite (Agali) & $\mathrm{U}-\mathrm{Pb}$ Zircon & $\begin{array}{l}2545 \pm 56 \\
2535 \pm 13\end{array}$ & Crystallization age & Santosh et al. (2013) \\
\hline $\begin{array}{l}\text { Trondhjemite } \\
\text { (Agali) }\end{array}$ & $\mathrm{U}-\mathrm{Pb}$ Zircon & $2547 \pm 7.4$ & Crystallization age & -do- \\
\hline \multicolumn{5}{|l|}{ Madurai Block } \\
\hline \multirow[t]{2}{*}{$\begin{array}{l}\text { Meta granite } \\
\text { (Kodaikanal) }\end{array}$} & $\begin{array}{l}\mathrm{U}-\mathrm{Pb} \text { zircon } \\
\text { evaporation }\end{array}$ & $2436 \pm 4$ & Magmatism & Bartlett et al. (1998) \\
\hline & $\begin{array}{l}\text { Younger zircon over } \\
\text { growths }\end{array}$ & $\sim 550$ & Metamorphism & - do- \\
\hline $\begin{array}{l}\text { Syntectonic } \\
\text { granites, (south of } \\
\text { KKPT shear zone) }\end{array}$ & $\begin{array}{l}\text { U-Pb Zircon and } \\
\text { Monazite }\end{array}$ & $\sim 790,600$ and 570 & $\begin{array}{l}\text { Neoproterozoic } \\
\text { thermotectonic } \\
\text { event(s) }\end{array}$ & $\begin{array}{l}\text { Ghosh et al. (1998, } \\
\text { 2004) }\end{array}$ \\
\hline $\begin{array}{l}\text { Post-tectonic } \\
\text { granitoids (south } \\
\text { of KKPT shear } \\
\text { zone) }\end{array}$ & $\begin{array}{l}\text { U-Pb Zircon and } \\
\text { Monazite }\end{array}$ & $\sim 525,550$ & $\begin{array}{l}\text { Neoproterozoic } \\
\text { metamorphism }\end{array}$ & -do- \\
\hline $\begin{array}{l}\text { Felsic } \\
\text { gneiss(Kadavur) }\end{array}$ & $\mathrm{U}-\mathrm{Pb}$ zircon & $766 \pm 8$ & $\begin{array}{l}\text { Intrusion age } \\
\text { Magmatism }\end{array}$ & Teale et al. (2011) \\
\hline $\begin{array}{l}\text { Vanji nagaram } \\
\text { granite }\end{array}$ & $\begin{array}{l}\mathrm{Rb}-\mathrm{Sr} \text { whole rock } \\
\text { isochron age }\end{array}$ & $620 \pm 43$ & Emplacement age & Nathan et al. (2001) \\
\hline Usilampatti Granite & $\begin{array}{l}\mathrm{Rb}-\mathrm{Sr} \text { whole rock } \\
\text { isochron age }\end{array}$ & $823 \pm 38$ & Emplacement age & Pandey et al. (2005) \\
\hline NW Madurai & $\begin{array}{l}\mathrm{Rb}-\mathrm{Sr} \text { whole rock } \\
\text { isochron age }\end{array}$ & $837 \pm 34$ & Emplacement age & Pandey et al. (1994) \\
\hline $\begin{array}{l}\text { Pudukkottai Pink } \\
\text { granite }\end{array}$ & $\begin{array}{l}\mathrm{Rb}-\mathrm{Sr} \text { whole rock } \\
\text { isochron age }\end{array}$ & $563.3 \pm 28$ & Emplacement age & $\begin{array}{l}\text { Chandra Sekaran et al. } \\
\text { (2016) }\end{array}$ \\
\hline Munnar granite & $\mathrm{U}-\mathrm{Pb}$ dating & $\begin{array}{l}740 \pm 30 \\
505 \pm 56\end{array}$ & Emplacement age & Odom (1982) \\
\hline Melur granite & $\mathrm{Rb}-\mathrm{Sr}$ dating & $795 \pm 17$ & Emplacement age & Nathan et al. (2001) \\
\hline Granite (Theni) & $\mathrm{U}-\mathrm{Pb}$ zircon & $722 \pm 11$ & Emplacement age & Plavsa et al. (2012) \\
\hline $\begin{array}{l}\text { Granite } \\
\text { (Kotamangalam) }\end{array}$ & $\mathrm{U}-\mathrm{Pb}$ zircon & $567 \pm 2$ & Protolith age & Ghosh et al. (2004) \\
\hline $\begin{array}{l}\text { Aklai granite (Sita- } \\
\text { parappanallur) }\end{array}$ & $\mathrm{U}-\mathrm{Pb}$ zircon & $662 \pm 20$ & Protolith age & Santosh et al. (2017) \\
\hline \multicolumn{5}{|l|}{ Trivendrum Block } \\
\hline Augen gneiss & U-Pb Zircon \& & $605 \pm 37$ & Emplacement age & Braun et al. (1998) \\
\hline Granitic pegmatite & Monazite $\mathrm{Th}-\mathrm{U}-\mathrm{Pb}$ & $\sim 470$ & Emplacement age & -do- \\
\hline Granite & $\begin{array}{l}\text { Flourapatite and } \\
\text { Monazite }\end{array}$ & $509 \pm 25$ & Emplacement age & $\begin{array}{l}\text { Berger and Braun } \\
\quad(1997)\end{array}$ \\
\hline $\begin{array}{l}\text { Pathanapuram } \\
\text { granite }\end{array}$ & ${ }^{207} \mathrm{~Pb} /{ }^{204} \mathrm{~Pb}$ & $534 \pm 5$ & Protolith age & Santosh et al. (2005) \\
\hline
\end{tabular}


and Kottamangalam areas. Neoarchean to Paleoproterozoic granites were described by several workers in the Northern Block (Balasubrahmanyan et al. 1979; Krogstad and Hanson 1988; Friend and Nutman 1991; Peucat et al. 1993, 2013). The tonalitic to trondhjemitic granite gneisses and charnockites from the Krishnagiri area in the north of Moyar-Bhavani described their protoliths age of 2550-2530 \pm 5 Ma (Peucat et al. 1993). However, the granitic rocks occurred near transition zone around Krishnagiri in the northern part of Tamil Nadu, described the age range from 3400 to 1000 Ma (Monard 1983). Similarly, in the Salem Block, $2528 \pm 2$ Ma granites gneiss and $\sim 2650 \mathrm{Ma}$ granites at Salem, $2647 \pm 11 \mathrm{Ma}$ granites at Kanjamalai, $2511 \pm 28 \mathrm{Ma}$ enderbite gneiss in Nilgiri block of Neoarchean ages were reported (Kohler and Srikantappa 1996; Ghosh et al. 2004; Sato et al. 2011). Some of the Paleoproterozoic granitoids are also described in the Northern Block, $\sim 2221 \mathrm{Ma}$ - Ebbari Malai, $\sim 2302 \mathrm{Ma}$ - Sholingar, $\sim 2254 \mathrm{Ma}$ - Gingee and $\sim 2254 \mathrm{Ma}$ - Tirukovilur granite (Balasubrahmanyan et al. 1979; Krogstad and Hanson 1988). The granites of the present study belong to two distinct age groups of Neoarchean-Paleoproterozoic and Neoproterozoic. The granites of Northern block including Veppanapalli, Vaniyambadi, Jakkampatty, and Bargur from the present study are possible equivalents of Krishnagiri of Neoarchean emplacements. Their emplacement sequence appears to be related the Neoarchean subduction-collision and accretionary tectonics (Saitoh et al. 2011; Yellappa et al. 2012; Santosh et al. 2012, 2013; Noack et al. 2013; Glorie et al. 2014; Samuel et al. 2014; Ratheesh Kumar et al. 2016) followed by crustal extension associated juvenile addition of magmas. In the Salem Block, the Neoproterozoic granites like Kullampatti granite is $534 \pm 15 \mathrm{Ma}$ and in Nilgiri block, the Ambalavayal granite of $595 \pm 20$ Ma, Kalpatta granite of $553 \pm 5 \mathrm{Ma}$ Peralimali granite of $750 \pm$ $40 \mathrm{Ma}$ are described by several workers (Santosh et al. 1986, 1989; Pandey et al. 1993; Miller et al. 1996). However, Neoproterozoic granitoids are predominantly described from the CSZ and Madurai Trivandrum Blocks. In the CSZ, the $\mathrm{Rb}-\mathrm{Sr}$ isotope and U-Pb zircon dating of leucocratic granites (trondhjemite) of Sankari, Tiruchengodu described as $534 \pm 15 \mathrm{Ma}$ (Rb-Sr isotope; Pandey et al. 1993) and $479 \pm 12 \mathrm{Ma}$, respectively (U-Pb zircon; Ghosh et al. 1996). The age of the Puliyampatti leucocratic granite is $471 \pm 10 \mathrm{Ma}$ (K-Ar GSI 1978) and that of Marudamalai granite is $619 \pm 35 \mathrm{Ma}(\mathrm{Rb}-\mathrm{Sr}$ isotope; Nathan et al. 2001). The A-type granite of Ayyermalai is described as $\sim 590 \mathrm{Ma}$ (U-Pb zircon; Sato et al. 2012). It is also reported that $819 \pm 26 \mathrm{Ma}$ age of Rapakivi granite at Tangalamvaripatti and $737 \pm 23 \mathrm{Ma}$ age of palgiogranite from Manamedu Ophiolite complex (Santosh et al. 2012) is within the CSZ. In the Madurai Block, the granite plutons range in age from 550 to 850 Ma. The Munnar granites are of $740 \pm 30 \mathrm{Ma}$ age (U-Pb zircon; Odom 1982), Pudukkottai granite is of $563 \pm 28 \mathrm{Ma}$ ( $\mathrm{Rb}-\mathrm{Sr}$ isotope; Chandra Sekaran et al. 2016), Usilampatti granite is of $823 \pm 38 \mathrm{Ma}$ (Rb-Sr; Pandey et al. 2005), Vanjinagarm granite is of $620 \pm 43 \mathrm{Ma}$, Melur granite is of $505 \pm 56 \mathrm{Ma}$ (Rb-Sr isotope; Nathan et al. 2001), Sitaparappanallur granite is of $722 \pm 11 \mathrm{Ma}$ (U-Pb zircon; Santosh et al. 2017) and Theni granite is of $795 \pm 17 \mathrm{Ma}$ (U-Pb zircon; Plavsa et al. 2012) as reported by earlier workers. Some of the Monazite ages of Vallamallai granite closer to Karur cluster around $500 \pm 2$ Ma has also been reported from this block. In the Trivandrum Block, the oldest zircons in the Pathanapuram granite pluton are in the range of 961-1149 Ma, with younger overgrowths at about 540-560 Ma, and at an age of $534 \pm 5 \mathrm{Ma}$, the Pathanapuram granite in the central domain of Achankovil shear zone was established (Santosh et al. 2005). The granite pegmatite of $470 \mathrm{Ma}$ and $509 \pm 25 \mathrm{Ma}$ granites from Ponmudi unit (Berger and Braun 1997) were also reported. All these age data indicates that the emplacement of alkali-granites and syenites in these tectonic blocks preceded calcalkaline felsic magmatism around $500 \mathrm{Ma}$ (Santosh et al. 1989, 2005) of Pan-African equivalents after the Neoptoerozoic subduction-collision and accretionary events.

There are also several reports of Pan-African related magmatism and associated lithologies within the CSZ and several parts of the SGT (Santosh and Drury 1988; Rajesh et al. 1996; Nathan et al. 2001; Braun 2006). The CSZ has also been interpreted as the zone of closure of the Neoproterozoic Mozambique Ocean (Collins and Pisarevsky 2005; Collins et al. 2006, 2007; Santosh et al. 2009) and a prolonged subduction-collisionaccretion history similar to Pacific-type of orogeny during Neoproterozoic-Cambrian periods along the craton-terrain margins (Santosh et al. 2009). Several imprints of Neoproterozoic arc related tectonics from the SGT, including oceanic remnants/ ocean plate stratigraphy and ultra-high temperature metamorphism (UHT) is described by several 

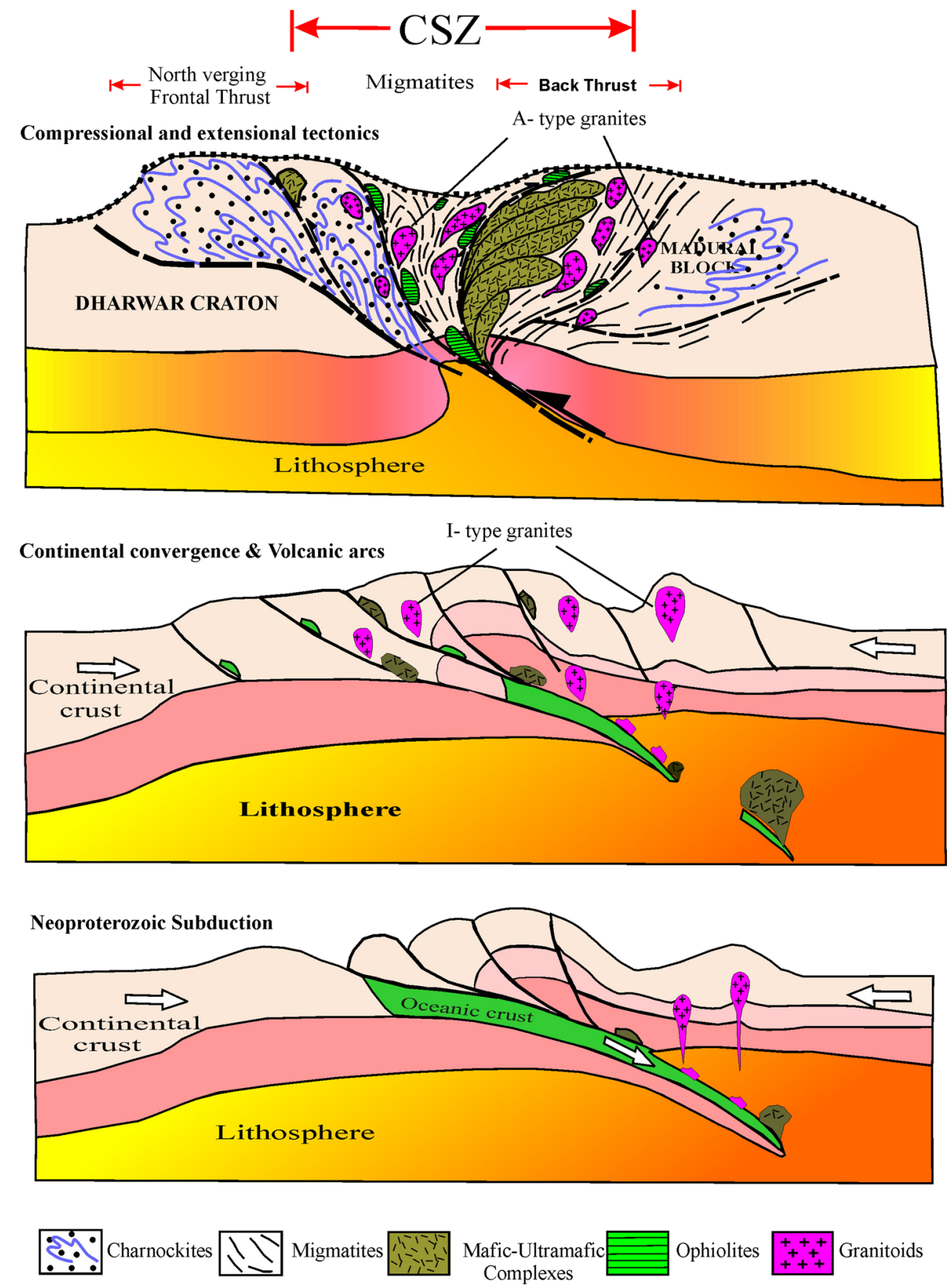

Figure 11. A schematic possible tectonic model showing Neoproterozoic subduction, continetal convergence and emplacement of volcanic arc to syn-collisional granites of I-type and emaplcements of A-type granites during extensional tectonics after post-collision.

workers (Tsunogae et al. 2008; Santosh et al. 2009, 2013; Yellappa et al. 2010). The occurrence of surpasubduction mechanism during Neoarchean and Neoproterozoic periods is also described based on the study of the Devanur Ophiolite complex, Agali Ophiolite complex, and Manamedu Ophiolite complex from the CSZ (Yellappa et al. 2010, 2012; Santosh et al. 2012, 2013). These tectonic events are correlated with major tectonic events in global scenario in terms of timing, deformation, magmatism, and metamorphism and evolution history. Recently, the occurrence of Neopreoterozoic subduction and associate magmatism (Santosh et al. 2017) and crustal extensional events (Brandt et al. 2014) in southern Madurai Block has also been described. Geochemical characteristics of 
studied granite plutons reveal I-type and A-type (figure $9 \mathrm{a}-\mathrm{b}$ ) with volcanic arc to syn-collisional and post-collisional signatures. I-type granitic magmatism probably associated with arc-continental collision of Neoproterozoic subduction (shown in figure 11). Their parental magma of these granitoids might have produced through mantle-crust source interaction which is typical of continental arc granities (Pearce 1996). The occurrence of A-type granites possibly related to anorogenic or transitional post-collision setting with largescale crustal extensional events resulting recycling of crustal sediments, melting of crustal rocks and juvenile addition of fluids led to the emplacement of several granites (figure 11). Similar tectonics and extensional related origin of A-granites are well described by several workers (Whalen et al. 1987; Rajesh et al. 1996; Rajesh 2000, 2008; Elbialy and Omar 2015). It is well known that basic and alkaline magmas related to extensional setting along the Mozambique suture of many segments including Malawi, southern Madagascar, southernmost India, Sri Lanka and part of Dronning Maud Land in Antarctica during the amalgamation between East and West Gondwana (Kroner et al. 1999; Collins et al. 2000). The high-temperature A-type granites, alkaline plutons and anorthosites, the high-temperature metamorphism of the granulites and the mantle-derived fluids associated with post-collisional extension along the Gondwana suture (Santosh and Yoshida 2001) have also been described.

Recent reviews on the formation of this Gondwana supercontinent suggest that the final amalgamation of Gondwana followed by the closure of the Mozambique Ocean, forming the East African orogen (Meert and Voo 1997; Kroner et al. 2000; Collins and Windley 2002; Meert 2003). Two PanAfrican orogenesis have been described, an older orogeny resulted from the collision of Eastern Africa with an ill-defined collage of continental blocks including parts of Madagascar, Sri Lanka, Seychelles, India and east Antarctica during the interval 750-620 Ma, which they referred to as East African orogeny (Meert 2003). The pre-PanAfrican signatures of south India, Madagascar and Sri Lanka are fundamentally important to characterize the Gondwana amalgamation tectonics around 500-550 Ma (McWilliams 1981; Unrug 1996; John et al. 2005). It is also described that several Pan-African domains/blets across the world expose upper to middle crustal levels and consist of ophiolites, subduction-collision related granitioids, island arc or passive margin assemblages similar to Phenorozoic plate tectonics (e.g., Arabian Nubian Shield - Arabia and northeast Africa, Damara Kaoko-Gariep Belt \& Luflin arc - South central and south-western Africa, West Congo Belt - Angola and Central Republic, Tan - Sahara Belt of West Africa etc., Kroner and Stern 2004). The widespread Neoproterozoic granitic activity in SGT and other parts of India can be correlated with the other crustal fragments of Eastern Gondwanaland (Rajesh et al. 1996). The Pan-African Orogeny and its related alkaline granitic magmatism is well established by several workers in SGT (Santosh et al. 1989; Rajesh et al. 1996; Rajesh and Santosh 1996). The geochemical characterstics and ages of many of the granite plutons are of Pan-African equivalents, and similar geochemical characterstics of granites are well described in Arabian Nubian Shield, Brazilian shield and several parts of the world (Jackson et al. 1984; Eyal et al. 2004; Kroner and Stern 2004). The structural geometry of Neoproterozoic ophiolite complex of Manamedu and tectonic history is also correlated with ophiolite settings of Arabian Nubian Sheild (Chetty et al. 2011).

\section{Conclusions}

- Petrological and geochemical characteristics of granitoids from the three distinct tectonic blocks of the southern granulite terrains show that they have feldspathic syenite to granite-ganodiorite compositions having metaluminous to slightly peraluminous nature.

- Geochemical characteristics of these granites suggest two distinct types of granitic magmatism: dominant I-type occurred along CSZ under continental volcanic arc settings to syncollisional setting and A-type, dominant in Madurai block, possibly in post-tectonic setting of extensional mechanism.

- The varied REE pattern of these granitoids suggests fractional crystallization. Trace element characteristics, REE and the tectonic discrimination diagrams reveal that they were derived from a predominant crustal source of deeper origin with variable degree of mantle inputs. The crystallization temperatures of the melts range from 700 to $800^{\circ} \mathrm{C}$, these lower temperatures might be the addition of more water or carbon dioxide during generation of melts.

- The available ages and the identified geochemical signatures of these granitoid rocks reveal 
that the emplacement of these plutons might be a complex, multiple-stage of evolution during Neoarchean and Neoproterozoic periods. In terms of age and geochemistry, the Neoproterozoic granites are well co-relatable with PanAfrican granites of Arabian Nubian Shield and Brazilian Shields.

\section{Acknowledgements}

The authors wish to thank the Director, NGRI, Hyderabad for the encouragement and support during the progress of the work. We are thankful to our colleagues Dr. A Keshava Krishna and Dr. M Satyanarayanan for their help in geochemical analysis. We are also very much thankful to anonymous reviewers for their helpful suggestions to improve the paper. This work also forms a part of Department of Science and Technology, Government of India, sponsored project (Ref: SR/FTP/ES-61/2014).

\section{References}

Balasubrahmanyan M N, Bhanumathi L, Bishni P K, Ray Barman T, Bhalla J K and Chandy K C 1979 A new age data of Gingee migmatite and its bearing on chronology of granulite terrain of Tamil Nadu; Unpubl. Geol. Surv. India P.R. for F.S. 1978-79.

Balasubrahmanyan M N and Sarkar A $1981{ }^{40} \mathrm{~K} /{ }^{40} \mathrm{Ar}$ ages of a suite of lower Palaeozoic post-tectonic granitoids, southern India; J. Geol. Soc. India 22 299-301.

Bartlett J M, Dougherty-Page J S, Harris N B W, Hawkesworth C J and Santosh M 1998 The application of single Harris zircon evaporation and model Nd ages to the interpretation of polymetamorphic terrains: An example from the Proterozoic mobile belt of south India; Contrib. Mineral. Petrol. 131 181-195.

Batchelor R A and Bowden P 1985 Petrogenetic interpretation of granitoid rocks using multicationic parameters; Chem. Geol. 48 43-55.

Berger $\mathrm{M}$ and Braun I $1997 \mathrm{~Pb}-\mathrm{Pb}$ dating of apatite by stepwise dissolution technique; Chem. Geol. 142 23-40.

Bhadra S and Nasipuri P 2017 Tectonothermal evolution of a garnet-bearing quartzo feldspathic gneiss from the Moyar shear zone, south India and its bearing on the Neoarchean accretionary tectonics; Lithos 274-275 1-18.

Bohm M, Van der Gugten C, Pin J L, Paquette I, Braun L M and Kriegsman 2003 Isotope geology and U-Pb geochronology of granites and high grade metamorphic rocks from the Achankovil unit, south India; Eur. J. Mineral. 1524.

Bonin B, Bebien J and Masson P 2002 Granite: A planetary point of view; Gondwana Res. 5 261-273.

Brandt S, Raith M M, Schenk V, Sengupta P, Srikantappa C and Gerdes A 2014 Crustal evolution of the Southern Granulite Terrane, south India: New geochronological and geochemical data for felsic orthogneisses and granites; Precamb. Res. 246 91-122.

Braun I, Montel J M and Nicollet C 1998 Electron probe dating of monazites from high-grade gneisses and pegmatites of the Kerala Khondalite Belt, southern India; Chem. Geol. 146 65-85.

Braun I 2006 Pan-African granitic magmatism in the Kerala Khondalite Belt, southern India; J. Asian Earth Sci. 28 $38-45$.

Brown M 1994 The generation, segregation, ascent and emplacement of granite magma: The migmatite-tocrustally-derived granite connection in thickened orogens; Earth Sci. Rev. 36 83-130.

Burg J P and Ford M 1997 Orogeny through time: An overview; Geol. Soc. London Spec. Publ. 121 1-17.

Castro A, Ferandez C and Vigneresse J L 1999a (eds) Understanding the granites: Integrating new and classical techniques; Geol. Soc. London Spec. Publ. 168.

Castro A, Patino Douce A E, Corretg L G, De la Rosa J D, El-Biad M and El-Hmidi H 1999b Origin of peraluminous granites and granodiorites, Iberian massif, Spain: An experimental test of granite petrogenesis; Contrib. Mineral. Petrol. 135 255-276.

Cenki B and Kriegsman L M 2005 Tectonics of the Neoproterozoic Southern Granulite Terrain, south India; Precamb. Res. 138 37-56.

Chacko T, Kumar G R R and Newton R C 1987 Metamorphic P-T conditions of the Kerala (south India) Khondalite Belt: A granulite-facies supracrustal terrain; J. Geol. 95 343-358.

Chandrasekharan C G 1996 Petrochemistry and origin of the granitic rocks in the amphibolite-granulite facies transition zone near Kaveripattanam, Dharmapuri district, Tamil Nadu; Ind. Mineral. 50(1) 63-72.

Chandrasekaran C G and Subrmanian S 1996 Petrogenesis of granitic rocks of low- to high-grade transition zone of Krishnagiri, Dharmapuri district, Tamil Nadu; Curr. Sci. 71(1) $42-48$.

Chandrasekharan C G 2002 Source characterstics and tectonic setting of granitoids of the Krishnagiri and Kaveripatnam areas of Dharmapuri Districts, Tamil Nadu; Ind. Mineral. 56 239-250.

Chandra Sekaran M, Bhutani R and Balakrishnan S 2016 $\mathrm{Rb}-\mathrm{Sr}$ and $\mathrm{Sm}-\mathrm{Nd}$ study of granite-charnockite association in the Pudukkottai region and the link between metamorphism and magmatism in the Madurai Block; $J$. Earth Syst. Sci. 125(3) 605-622.

Chappell B W and White A J R 1974 Two contrasting granite types; Pacific Geol. 8 173-174.

Chappell B W and White A J R 1992 I- and S-type granites in the Lachlan fold belt; Trans. Roy. Soc. Edinburgh (Earth Sci.) 83 1-16.

Chetty T R K and Bhaskar Rao Y J 2006 The Cauvery Shear Zone, Southern Granulite Terrain, India: A crustal-scale flower structure; Gondwana Res. 10 77-85.

Chetty T R K, Bhaskar Rao Y J and Narayana B L 2003 A structural cross section along Krishnagiri-Palani corridor, Southern Granulite Terrain of India. Tectonics of Southern Granulite Terrain Kuppam-Palani Geotransect (ed.) Ramakrishna M, Geol. Soc. India Memoir 50 255-278.

Chetty T R K, Yellappa T, Nagesh P, Mohanty D P, Venkatasivappa V, Santosh M and Tsunogae T 2011 Structural 
anatomy of a dismembered ophiolite suite from Gondwana: The Manamedu complex, Cauvery suture zone, southern India; J. Asian Earth Sci. 42 176-190.

Chetty T R K, Yellappa T and Santosh M 2016 Crustal architecture and tectonic evolution of the Cauvery Suture Zone, southern India; J. Asian Earth Sci. 130 166191.

Clark D B, Mac Donald M A and Tate M C 1997 Late Devonian mafic-felsic magmatism in the Meguma zone, Nova Scotia; The nature of magmatism in the appalachian orogeny (eds) Krishan Sinha A, Joseph B Whalen and Jhon P Hogan, pp. 107-128.

Clark C, Collins A S, Kinny P D, Timms N E and Chetty T R K 2009 SHRIMP U-Pb age constraints on the age of charnockite magmatism and metamorphism in the Salem Block, southern India; Gondwana Res. 16 27-36.

Collins A S 2003 Structure and age of the Leeuwin Complex, Western Australia: Constraints from field mapping and U-Pb isotope analysis; Austr. J. Earth Sci. 50 585-599.

Collins W J and Sawyer E W 1996 Pervasive granitoid magma transfer through the lower-middle crust during non-coaxial compressional deformation; J. Metamor. Geol. 14(5) 565-579.

Collins A S and Windley B F 2002 The tectonic evolution of central and northern Madagascar and its place in the final assembly of Gondwana; J. Geol. 110 325-340.

Collins A S and Pisarevsky S A 2005 Amalgamating eastern Gondwana: The evolution of the Circum-Indian orogens; Earth Sci. Rev. 71 229-270.

Collins W J, Bearns S D, White A J R and Cheppal B W 1982 Nature and origin of A-type granites with particular reference to southern Australia; Contrib. Mineral. Petrol. 10 189-200.

Collins A S, Razakamanana T and Windley B F 2000 Neoproterozoic extensional detachment in central Madagascar: Implications for the collapse of the East African orogen; Geol. Mag. 137 39-51.

Collins A S, Santosh M, Kropinski L, Hand M P, Clark C, Sajeev K, McKenzie S and Kinny P D 2006 The Mozambique ocean suture in southern India: Age and significance of granulites in the Palghat-Cauvery Shear Zone System; Presented at AESC2006 Melbourne, Australia.

Collins A S, Clark C, Sajeev K, Santosh M, Kelsey David E and Martin H 2007 Passage through India: Mozambique Ocean suture, high pressure granulites and PalghatCauvery shear zone system; Terra Nova 19 41-147.

Collins A S, Clark C and Plavsa D 2014 Peninsular India in Gondwana: Tetectonothermal evolution of the Southern Granulite Terrain and its Gondwana counterparts; Gondwana Res. 25 190-203.

Condie K C 1973 Archean magmatism and crustal thickening; Geol. Soc. Am. Bull. 84 2981-2291.

Condie K, Allen P and Narayana B L 1982 Geochemistry of the Archean low- to high-grade transition zone, south India; Contrib. Mineral. Petrol. 81 157-167.

Condie K C, Bowling G P and Allen P 1986 Origin of granites in an Archean high-grade terrain, south India; Contrib. Mineral. Petrol. 92 93-103.

Condie K C and Kroner A 2013 The building blocks of continental crust: evidence for a major change in the tectonic setting of continental growth at the end of the Archean; Gondwana Res. 23 394-402.
David C C and Chappell B W 1992 Petrogenesis of felsic I-type granites: An example from northern Queensland; Geol. Soc. Am. Spec. Paper 83 115-114.

De La Roche H, Laterrier J G, Caude P and Marchal M 1980 A classification of volcanic rocks using R1-R2 diagram and major element analyses - its relationships and current nomenclature; Chem. Geol. 29 183-210.

Dhanaraju R, Varma H M, Padmanabhan N and Mahadevan T M 1983 I-type and S-type classification of the Precambrian granitoids of southern India and its possible relevance to mineral exploration; In: Precambrian of South India (eds) Naqvi S M and Rogers J J W, Geol. Soc. India Memoir, pp. 389-400.

Drury S A, Harris N B W, Holt R W, Reeves Smith G J and Wightman R T 1984 The Precambrian tectonics and crustal evolution in south India; J. Geol. 92 3-20.

Eby G N 1992 Chemical subdivision of the A-type granitoids: Petrogenetic and tectonic implications; Geology $20641-$ 644.

Ellis D J and Thompson A B 1986 Sub-solidus and partial melting reactions in the quartz-excess $\mathrm{CaO}+\mathrm{MgO}+$ $\mathrm{Al}_{2} \mathrm{O}_{3}+\mathrm{SiO}_{2}+\mathrm{H}_{2} \mathrm{O}$ system under water-excess waterdeficient conditions to $10 \mathrm{~kb}$ : Some implications for the origin of peraluminous melts from mafic rocks; J. Petrol. 27 91-121.

El-bialy M Z and Omar M M 2015 Spatial association of Neoproterozoic continental arc-type and post-collision A-type granitoids in the Arabian-Nubian Shield: The Wadi AlBaroud older and Younger granites, Northeastern Desert, Egypt; J. Afric. Earth Sci. 103 1-29.

Eyal M, Litvinovsky B A, Katzir Y and Zanvilevich A N 2004 The Pan-African high-K calc-alkaline peraluminous Elat granite from southern Israel: Geology, geochemistry and petrogenesis; J. Afric. Earth Sci. 40 115-136.

Friend C R L and Nutman A P 1991 SHRIMP U-Pb geochronology of the Closepet granite and peninsular gneisses, Karnataka, south India; J. Geol. Soc. India 38 $357-368$.

Friend C R L and Nutman A P 1992 Response of U-Pb isotopes and whole rocks geochemistry to $\mathrm{CO}_{2}$ induced granulite metamorphism, Kabbaldurga, Karnataka, South India; Contrib. Mineral. Petrol. 111 299-310.

Frost B R, Barnes C G, Collins W J, Arculus R J, Ellis D J and Frost C D 2001 A geochemical classification for granitic rocks; J. Petrol. 42 2033-2048.

Geological Survey of India 1978 Geological Survey of India initiates Potassium-Argon dating; Geol. Surv. India Rev., Central Headquarters, Calcutta.

Geological Survey of India 2009 Global correlation of Atype granites and related rocks, their mineralization, and significance in lithosperhic evolution; International Geoscience Programme (IGCP-510) 19-20 March 2010, GSI, Nagpur.

Ghani A A, Searle M, Robb L and Chung S L 2013 Transitional I-S type characteristic in the Main Range Granite, Peninsular Malaysia; J. Asian Earth Sci. 76 225240.

Ghosh Subhasish, Balasubramanian E, Nathan N P, Kanishkan B and Krishnan V 1996 Rb-Sr isotopic studies and geochemistry of granitoids and charnockite from Tamil Nadu; Geol. Surv. India Unpubl. Progress Report F.S. 1992-93. 
Ghosh J G, Zartman R E and de Wit M J 1998 Reevaluation of tectonic framework of southernmost India: New $\mathrm{U}-\mathrm{Pb}$ geochronological and structural data, and their implication for Gondwana reconstruction; J. Afric. Earth Sci. $2785-86$.

Ghosh J G, Maarten De wit R E and Zartman R E 2004 Age and tectonic evolution of neoproterozoic ductile shear zone in Southern Granulite Terrain, India, with implications for Gondwana studies; Tectonics $\mathbf{2 3}$ TC3006, https://doi. org/10.1029/2002 2004 .

Glorie S, De Grave J, Singh T, Payne J L and Collins A S 2014 Crustal root of the Eastern Dharwar Craton: Zircon $\mathrm{U}-\mathrm{Pb}$ age and $\mathrm{Lu}-\mathrm{Hf}$ isotopic evolution of the East Salem Block, southeast India; Precamb. Res. 249 229-246.

Gopalakrishnan K 1994 An overview of southern granulites terrain of Tamil Nadu: Constraints in reconstruction of Precambrian assembly of Gondwanaland; Gondwana $9^{\text {th }}$ Int. Gondwana Symp., Oxford \& IBH Publications Co. Ltd., 2, 1003-1026.

Harris N B W, Santosh M and Taylor P N 1994 Crustal revolution in south India: Constraints from Nd-isotopes; J. Geol. 102 139-150.

Hussain S M, Narayana B L and Naqvi S M 1996 Plagiogranitic differentiates from ultramafic-mafic-felsic suite along Cauvery suture at Manamedu in the granulite terrain of south India; Proc. of IX Convention of Indian Geology Congress 1996, pp. 155-160.

Jackson N J, Walsh J N and Pegram E 1984 Geology, geochemistry and petrogenesis of late Precambrian granitoids in the Central Hijaz region of the Arabian Shield; Contrib. Mineral. Petrol. 87 205-219.

Jacobs J, Bauer W and Fanning C N 2003 Late Neoproterozoic/early Paleozoic events in central Droning Maud Land and significance for the southern extension of the East African orgen into East Antarctica; Precamb. Res. $12627-53$.

Janardhan A S, Jayananda M and Shankara M A 1994 Formation and tectonic evolution of granulites from the Biligirirangan and Nilgiri hills, south India: Geochemical and isotopic constraints; J. Geol. Soc. India 44 27-40.

John M, Manish Balakrishnan S and Bhadra B K 2005 Contrasting metamorphism across Palghat-Cauvery Shear Zone, south India; J. Earth Syst. Sci. 114(2) 143158.

Kohler H and Srikantappa C 1996 Age and origin of enderbites from the Nilgiri Hills massif, S. India; V.M. Goldschmidt Conf. March $31^{\text {st }}-$ April $4^{\text {th }}$, 1996, Heidelberg, Germany.

Koizumi T, Tsunogae T, Santosh M, Tsutsumi Y, Chetty T R K and Saitoh Y 2014 Petrology and zircon U-Pb geochronology of metagabbros from a mafic-ultramafic suite at Aniyapuram: Neoarchean to Early Paleoproterozoic convergent margin magmatism and Neoproterozoic high-grade metamorphism in southern India; J. Asian Earth Sci. 95 51-64.

Kriegsman L M 1995 The Pan-African event in east Antarctica: A view from Sri Lanka and the Mozambique Belt; Precamb. Res. 75 263-277.

Krishna A K, Murthy M N and Govil P K 2007 Multielement analysis of soils by wave length-dispersive X-ray fluorescence Spectrometry; Atomic Spectr. 28(6) 202214 .
Krogstad K J and Hanson G N 1988 U-Pb ages and Sr$\mathrm{Pb}$ and $\mathrm{Nd}$-Isotope data gneisses near the Kolar Schist belt: Evidences for the juxtaposition of discrete Archean Terrains; J. Geol. Soc. India 3(1) 60-62.

Kroner A 1980 Pan-African crustal evolution; Episodes 2 3-8.

Kroner A and Stern R J 2004 Pan-African orogeny; Encyclopedia of Geology, vol. 1, Elsevier, Amsterdam.

Kroner A, Windley B F, Jaeckel P, Brewer T S and Razakamanana T 1999 New zircon ages and regional significance for the evolution of the Pan-African orogen in Madagascar; J. Geol. Soc. London 156 1125-1135.

Kroner A, Hegner E, Collins A S, Windley B F, Brewer T S, Razakamanana T and Pidgeon R T 2000 Age and magmatic history of the Antananarivo Block, Central Madagascar, as derived from zircon geochronology and Nd isotope systematics; Am. J. Sci. 330 251-258.

Kusky T M, Abdelsalam M, Stern R J and Tucker R D 2003 Evolution of the East African and related orogens and the assembly of Gondwana; Precamb. Res. 123 82-85.

Mallikharjuna Rao J, Suresh Raj T and Rama Rao 2005 Petrology and geochemistry of Puliampatti and PollachiUdumalpet granites of southern granulite terrain and their tectonic implications; J. Appl. Geochem. 7256 270.

Maniar P D and Piccoli P M 1989. Tectonic discrimination of granitoids; Geol. Soc. Am. Bull. 101 635-643.

McWilliams M O 1981 Paleomagnetism and Precambrian tectonic evolution; In: Precambrian plate tectonics (ed.) Kroner A, Amsterdam; Elsevier, pp. 642-687.

Meert J 2003 A synopsis of events related to the assembly of eastern Gondwana; Tectonophys. 362 1-40.

Meert J G and Van der Voo R 1997 The assembly of Gondwana 800-550 Ma; J. Geodyn. 23 223-236.

Miller J S, Santosh M, Pressley R A, Clemens A S and Rogers J J W 1996 A Pan-African thermal event in southern India; J. South East Asian Earth Sci. 14 127-136.

Meissner B, Deters P, Srikatappa C and Kohler H 2002 Geochronological evolution of the Moyar, Bhavani and Palghat shear zones of southern India: Implications for east Gondwana correlations; Precamb. Res. 114149 175.

Monard J R 1983 Evolution of sialic terrains in the vicinity of the Holenarsipur belt, Hassan District, Karnataka, India; In: Precambrian South India (eds) Naqvi S M and Rogers J J W, Geol. Soc. India Memoir 4 343-364.

Naqvi S M and Rogers J J W 1987 Precambrian geology of India; Oxford University Press, New York, 223p.

Nathan N P, Krishna Rao A V, Bhalla J K, Balasubramanian E, Subramanian N, Oberoi L K, Natarajan V, Gopalakrishna K and Raman R 1994 Geochemistry and geochronology of the pegmatoidal granite of Sankari-Tiruchengodu area, Tamil Nadu; Ind. Mineral. 48 113-123.

Nathan N P, Balasubramanian E, Ghosh S and Ray Barman T 2001 Neoproterozoic acid magmatism in Tamil Nadu, south India: Geochemical and geochronological constituents; Gondwana Res. 4 714-715.

Noack N M, Kleinschrodta R, Kirchenbaura M, Fonsecab R O C and Munker C $2013 \mathrm{Lu}-\mathrm{Hf}$ isotope evidence for Paleoproterozoic metamorphism and deformation of Archean oceanic crust along the Dharwar Craton margin, southern India; Precamb. Res. 233 206-222. 
O'Connor J T 1965 A classification for quartz rich igneous rocks based on feldspar ratios; Proceeding Paper U.S. Geol. Surv. 525B 79-84.

Odom A L 1982 Isotope age determinations of rock and mineral samples from Kerala, India; Unpublished Final Report UN Case No 81-10084 KMEDP Trivandrum, 10.

Pandey B K, Krishna V, Sastry D V L N, Chabria T, Mary K K and Dhanaraju R 1993 Pan-African wholerock $\mathrm{Rb}-\mathrm{Sr}$ isochron ages for the granites and pegmatites of Kullampatti-Suriyamalai area, Salem District, Tamil Nadu, India; VI Nat. Symp. Mass spectrometry, Ind. Inst. Petroleum, Dehradun, Abst. vol., pp. 480-482.

Pandey U K, Chabria T and Veena Krishnamurthy P 1994 $\mathrm{Rb}-\mathrm{Sr}$ geochronology of late Proterozoic A-type granites in parts of Madurai district, Tamil Nadu: Implications on uranium, rare earth, rare metal distributions; J. Atomic Mineral. Sci. 2 79-87.

Pandey U K, Pandey B K and Krishnamurthy P 2005 Geochronology (Rb-Sr, Sm-Nd and $\mathrm{Pb}-\mathrm{Pb}$ ) of the Proterozoic granulitic and granitic rocks around Usilampatti, Madurai District, Tamil Nadu: Implications on age of various lithounits; J. Geol. Soc. India 66 539-551.

Paquette J L, Nédélec A, Moine B and Rakotondrazafy M $1994 \mathrm{U}-\mathrm{Pb}$ single zircon $\mathrm{Pb}$ evaporation and $\mathrm{Sm}-\mathrm{Nd}$ isotopic study of a granulite domain in SE Madagascar; Earth Planet. Sci. Lett. 155 45-56.

Patino Douce A E 1997 Generation of metaluminous A-type granites by low-pressure melting of calc-alkaline granitoids; Geology 25(8) 743-746.

Patino Douce A E 1999 What do experiments tell us about the relative contributions of crust and mantle to the origin of granitic magmas? In: Understanding granites: Integrating new and classical techniques (eds) Castro A, Fernandez C and Vigneressc J L, Geol. Soc. London Spec. Publ. 168 55-75.

Patino Douce A E and Harris N 1998 Experimental constrains on Himalayan anatexis; J. Petrol. 39 698-710.

Pearce J A, Harris N B W and Tindle A G 1984 Trace element discrimination diagrams for the tectonic interpretation of granitic rocks; J. Petrol. 25 956-983.

Pearce J A 1996 Source and setting of granitic rocks; Episodes 19 120-125.

Peucat J J, Vidal P, Bernard-Gmffiths J and Conde K C $1989 \mathrm{Sr}, \mathrm{Nd}$ and $\mathrm{Pb}$ isotopic systems in the Archean low- to high-grade transition zone of southern India: Synaccretion vs. post-accretion of granulites; J. Geol. 97 $537-550$

Peucat J J, Mahabhaleswar B and Jayananda M 1993 Age of younger tonalitic magmatism and granulite metamorphism in the south India transition zone (Krishnagiri area): Comparision with older peninsular gneisses from Hassan-Gorur area; J. Metamor. Geol. 11 879888.

Peucat J J, Jayananda M, Chardon D, Capdevilla R, Fanning C M and Paquette J L 2013 The lower crust of the Dharwar Craton, southern India: Patchwork of Archean granulitic domains; Precamb. Res. 227 4-28.

Pitcher W S 1993 The nature and origin of Granites; Chapman and Hall, London.

Plavsa D, Collins A S, Foden J F, Kropinski L, Santosh M, Chetty T R K and Clark C 2012 Delineating crustal domains in peninsular India: Age and chemistry of orthopyroxene-bearing felsic gneisses in the Madurai Block; Precamb. Res. 198-199 77-93.

Ragland P C 1989 Basic analytical petrology; Oxford University Press, New York.

Rai S S, Srinagesh D and Gaur V K 1993 Granulite evolution in south India: A seismic tomographic prospective; Geol. Soc. India Memoir 25 235-264.

Rajesh H M 2000 Characterization and origin of compositionally zoned aluminous A-type granite form south India; Geol. Mag. 137(3) 291-318.

Rajesh H M 2004 The igneous charnockite-high-K alkalicalcic I-type granite-incipient charnockite association in Trivandrum Block, southern India: Contrib. Mineral. Petrol. 147 346-362.

Rajesh H M 2008 Petrogenesis of two granites from the Nilgiri and Madurai Blocks, southwestern India: Implications for charnockite-calc-alkaline granite and charnockitealkali (A-type) granite link in high-grade terrains; Precamb. Res. 162(1) 180-197.

Rajesh H M and Santosh M 1996 Alkaline magmatism in Peninsular India; In: The Archean and Proterozoic terrains in southern India within East Gondwana (eds) Santosh M and Yoshida M, Gondwana Res. Memoir $\mathbf{3}$ 91-115.

Rajesh H M, Santosh M and Yoshida M 1996 The felsic magmatic province in East Gondwana: Implications for Pan-African tectonics; J. Southeast Asian Earth Sci. 14(3-4) 275-291.

Ramakrishnan M 1991 Evolution of the high-grade terrains of southern India and Sri Lanka; Proceedings (Abstract) of symposium on "Composition and evolution of highgrade gneiss terrains", Kandy, Sri Lanka, pp. 57-59.

Ramakrishnan M 2003 Craton-mobile belt relations in southern granulite terrain; Geol. Soc. India Memoir 50 1-24.

Ramakrishnan M and Vaidyanathan 2008 Geology of India; Vol. 1, Geol. Soc. India, Bangalore.

Ratheesh Kumar R T, Santosh M, Yang Q Y, Ishwar-Kumar C, Song Chen N and Sajeev K 2016 Archean tectonics and crustal evolution of the Biligiri Rangan Block, southern India; Precamb. Res. 275 406-428.

Roy M and Dhanaraju R 1999 Petrogenetic model of A-type granitoids of the Kullampatti area, Salem district, Tamil Nadu, India; Gondwana Res. 2(1) 127-135.

Roy P, Balaram V, Satyanarayan M and Gnaneswara Rao T 2007 New REE and trace element data on two kimberlite reference materials by ICP-MS; Geost. Geoanal. Res. 31 261-273.

Rundick R L 1991 Restites, Eu anomalies and the lower continental crust; Geoch. Cosmoc. Act. 56 963-970.

Rushmer T, Brown M and Bergantz G 1998 Penrose Conference Report: Processes of crustal differentiation: Crust-mantle interactions, melting and granite migration through the crust; July 4-11, 1998.

Saitoh Y, Tsunoga T, Santosh M, Chetty T R K and Horie K 2011 Neoarchean high-pressure metamorphism from the northern margin of the Palghat-Cauvery Suture Zone, southern India: Petrology and zircon SHRIMP geochronology; J. Asian Earth Sci. 42(3) 268-285.

Samuel V O, Santosh M, Liu S, Wang W and Sajeev K 2014 Neoarchean continental growth through arc magmatism in the Nilgiri Block, southern India; Precamb. Res. 245 146-173. 
Santosh M 1996 The Trivandrum and Nagercoil granulite blocks; In: The Archean and Proterozoic terrains of southern India within East Gondwana (eds) Santosh M and Yoshida M, Gondwana Res. Memoir 3 243-278.

Santosh M and Nair N G K 1983 Petrochemistry of the Chenganoor granite, Alleppey district, Kerala; J. Geol. Soc. India 24 291-298.

Santosh M and Drury S A 1988 Alkali granites with PanAfrican affinities from Kerala, south India; J. Geol. 96 616-626.

Santosh M and Sajeev K 2006 Anticlockwise evolution of ultrahigh-temperature granulites within continental collision zone in southern India; Lithos 92 447-464

Santosh M, Nair N G K, Pandey K and Gopalan K 1986 $\mathrm{Rb}-\mathrm{Sr}$ geochronology of the Ambalavayal granite, Kerala; J. Geol. Soc. India 27 309-312.

Santosh M, Iyer S S, Vasconcellos M B A and Enzweiler J 1989 Late Precambrian alkaline plutons in southwest India: Geochronological and rare-earth element constraints on Pan-African magmatism; Lithos 24 65-79.

Santosh M, Rajan P K and Nair N G K 1993 The Pariyaram granite, Trichur district, Kerala - its petrochemistry; Bull. Indian Geol. Assoc. 16 33-43.

Santosh M and Yoshida M 2001 Pan-African extensional collapse along the Gondwana Suture; Gondwana Res. 4(1) $188-191$

Santosh M, Tanaka T, Yokoyama K and Collins A S 2005 Late Neoproterozoic-Cambrian felsic magmatism along transcrustal shear zones in southern India: $\mathrm{U}-\mathrm{Pb}$ electron microprobe ages and implications for the amalgamation of the Gondwana Supercontinent; Gondwana Res. 8 31-42.

Santosh M, Maruyama S and Sato K 2009 Anatomy of a Cambrian suture in Gondwana: Pacific-type orogeny in southern India; Gondwana Res. 16(2) 321-341.

Santosh M, Xiao W J, Tsunogae T, Chetty T R K and Yellappa T 2012 The Neoproterozoic subduction complex in southern India: SIMS zircon U-Pb ages and implications for Gondwana assembly; Precamb. Res. 192-195 190208.

Santosh M, Shaji E, Tsunogae T, Ram Mohan M, Satyanarayanan M and Horie K 2013 Neoarchean suprasubduction zone ophiolite from Agali hill, southern India: Petrology, zircon SHRIMP U-Pb geochronology, geochemistry and tectonic implications; Precamb. Res. 231 301-324.

Santosh M, Yang Q Y, Shaji E, Tsunogae T, Ram Mohan M and Satyanarayanan M 2015 An exotic Mesoarchean microcontinent: The Coorg Block, southern India; Gondwana Res. 27(1) 165-195.

Santosh M, Yang Q Y, Shaji E, Ram Mohan M, Tsunogae T and Satyanarayanan M 2016 Oldest rocks from peninsular India: Evidence for Hadean to Neoarchean crustal evolution; Gondwana Res. 29(1) 105-135.

Santosh M, Nan Hu C, Fang He X, Shan Li S, Tsunogae T, Shaji E and Indu G 2017 Neoproterozoic arc magmatism in the southern Madurai Block, India: Subduction, delamination, continental outbuilding, and the growth of Gondwana; Gondwana Res. 45 1-42.

Sato K, Santosh M, Tsunogae T, Chetty T R K and Hirata T 2011 Laser ablation ICP mass spectrometry for zircon $\mathrm{U}-\mathrm{Pb}$ geochronology of metamorphosed granite from the Salem Block: Implication for Neoarchaean crustal evolution in southern India. J. Miner. Petrol. Sci. 106 1-12.
Sato K, Santosh M, Chetty T R K and Hirata T 2012 U-Pb zircon geochronology of granites and charnockites from southern India: Implications for magmatic pulses associated with plate tectonic cycles within a Precambrian suture zone; Geol. J. 47(2-3) 237-252.

Sawyer E W 1994 Melt segregation in the continental crust; Geology 22 1019-1022.

Shackelton R M 1986 Precambrian collision tectonics in Africa. In: Collision tectonics (eds) by Coward M P and Ries A C, Geol. Soc. London Spec. Publ. 19 329-349.

Sun S S and Nesbitt R W 1978 Petrogenesis of Archean ultrabasic and basic volcanics. Evidence from rare earth elements; Contrib. Contrib. Mineral. Petrol. 65 301-328.

Sun S S and Mc donough W F 1989 Chemical and isotopic systematics of oceanic basalts: Implications of mantle composition and processes; Geol. Geol. Soc. London Spec. Publ. 42 313-345.

Teale W, Collins A, Foden J, Payne J, Plavsa D, Chetty T R K, Santosh M and Fanning M 2011 Cryogenian ( $\sim 830 \mathrm{Ma})$ mafic magmatism and metamorphism in the northern Madurai Block, southern India: A magamtic link between Sri Lanka and Madagascar; J. Asian Earth Sci. $42223-233$.

Thampi P K, Nair P K R and Balasubramonian G 1993 Petrochemistry and tectonic evolution of Munnar granite, Idukki district, Kerala; Curr. Sci. 64 238-243.

Tsunogae T, Santosh M and Dubessy J 2008 Fluid characteristics of high- to ultrahigh-temperature metamorphism in southern India: A quantitative Raman spectroscopic study; Precamb. Res. 162 198-211.

Tuttle O F and Bowen N 1958. Origin of granite in the light of experimental studies in the system $\mathrm{NaAlSi}_{3} \mathrm{O}_{8}-\mathrm{KAlSi}_{3} \mathrm{O}_{8}-\mathrm{SiO}_{2}-\mathrm{H}_{2} \mathrm{O}$; Geol. Soc. Am. Memoir $\mathbf{7 4} 153$.

Unrug R 1996 The assembly of Gondwanaland land; Episodes $1911-20$.

Viswanathan T V, Gopalakrishnan K, Ganesan T M and Raman R 1990 Cauvery suture zone - its implications; Group Discussion on Suture Zones - Young and Old, Wadia Institute of Himalayan Geology, Geol. Surv. India Extended abstract, Dehradun, pp. 47-54.

Whalen J B, Currie K L and Chappell B W 1987 A-type granites: Geochemical characteristics, discrimination and petrogenesis; Contrib. Mineral. Petrol. 95 407-419.

Whalen J B, Jenner G A, Longstaffe F J, Gariépy C and Fryer B J 1997 Implications of granitoid geochemical and isotopic $(\mathrm{Nd}, \mathrm{O}, \mathrm{Pb})$ data from the Cambrian-Ordovician Notre Dame arc for the evolution of the Central Mobile belt, Newfound land Appalachians; Geol. Soc. Am. Memoir 191 367-395.

Windley B F, Razafiniparany A, Razakamanana T and Ackermund D 1994 Tectonic framework of the Precambrian of Madagascar and its Gondwana connections: A review and reappraisal; Geol. Rund. 83 642-659.

Yellappa T, Chetty T R K, Tsunogae T and Santosh M 2010. Manamedu complex: Geochemical constraints on Neoproterozoic suprasubduction zone ophiolite formation within Gondwana suture in southern India; J. Geodyn. 50268 285.

Yellappa T, Santosh M, Chetty T R K, Sanghoon Kwon, Chansoo Park, Nagesh P, Mohanty D P and Venkatasivappa V 2012 A Neoarchean dismembered 
ophiolite complex from southern India: Geochemical and geochronological constraints on its suprasubduction origin; Gondwana Res. 21 245-265.

Yellappa T, Venkatasivappa V, Koizumi T, Chetty T R K, Santosh M and Tsunogae T 2014 The

Corresponding editor: N V CHALAPATHI RAO mafic-ultramafic complex of Aniyapuram, Cauvery Suture Zone, southern India: Petrological and geochemical constraints for Neoarchean suprasubduction zone tectonics; J. Asian Earth Sci. 95 81-98. 